\title{
OPEN Metabolomics and transcriptomics to decipher molecular mechanisms underlying ectomycorrhizal root colonization of an oak tree
}

\author{
M. Sebastiana ${ }^{1 凶}$, A. Gargallo-Garriga ${ }^{2}$, J. Sardans ${ }^{3,4}$, M. Pérez-Trujillo ${ }^{5}$, F. Monteiro ${ }^{6,7}$, \\ A. Figueiredo ${ }^{1}$, M. Maia ${ }^{1,8}$, R. Nascimento ${ }^{1}$, M. Sousa Silva ${ }^{8}$, A. N. Ferreira ${ }^{8}$, C. Cordeiro ${ }^{8}$, \\ A. P. Marques ${ }^{8}$, L. Sousa ${ }^{9}$, R. Malhó ${ }^{1}$ \& J. Peñuelas ${ }^{3,4,10}$
}

Mycorrhizas are known to have a positive impact on plant growth and ability to resist major biotic and abiotic stresses. However, the metabolic alterations underlying mycorrhizal symbiosis are still understudied. By using metabolomics and transcriptomics approaches, cork oak roots colonized by the ectomycorrhizal fungus Pisolithus tinctorius were compared with non-colonized roots. Results show that compounds putatively corresponding to carbohydrates, organic acids, tannins, longchain fatty acids and monoacylglycerols, were depleted in ectomycorrhizal cork oak colonized roots. Conversely, non-proteogenic amino acids, such as gamma-aminobutyric acid (GABA), and several putative defense-related compounds, including oxylipin-family compounds, terpenoids and B6 vitamers were induced in mycorrhizal roots. Transcriptomic analysis suggests the involvement of GABA in ectomycorrhizal symbiosis through increased synthesis and inhibition of degradation in mycorrhizal roots. Results from this global metabolomics analysis suggest decreases in root metabolites which are common components of exudates, and in compounds related to root external protective layers which could facilitate plant-fungal contact and enhance symbiosis. Root metabolic pathways involved in defense against stress were induced in ectomycorrhizal roots that could be involved in a plant mechanism to avoid uncontrolled growth of the fungal symbiont in the root apoplast. Several of the identified symbiosis-specific metabolites, such as GABA, may help to understand how ectomycorrhizal fungi such as $P$. tinctorius benefit their host plants.

Most land plants live in association with specialized soil-born fungi that colonize their roots forming mycorrhizae, the most widespread plant-symbiotic relationship. At the centre of this symbiosis is the mutual exchange of nutrients between partners: the fungus provides mineral nutrients to the plant, that in turn transfers to the fungus sugars produced during photosynthesis ${ }^{1}$. Temperate forest trees, such as Pinus, Oaks and Eucalyptus form a distinct type of mycorrhizas, called ectomycorrhizas (ECM), with Basidiomycotic and Ascomycotic fungi, such as boletes and truffles. In ECMs, the fungal mycelium colonizes the apoplast of root cells forming a net of hyphae that surrounds the epidermal and/or the cortex cells. By also spreading into the soil, the fungal mycelium can access mineral nutrients very efficiently, such as nitrogen and phosphorous, which are then transferred to the root, ECM greatly increasing the absorptive surface area of the host plant root system. However, ECM symbiosis can also help plants to increase resistance to multiple stresses. Indeed, several studies point to

\footnotetext{
${ }^{1}$ Plant Functional Genomics Group, BiolSI - Instituto de Biosistemas e Ciências Integrativas, Faculdade de Ciências, Universidade de Lisboa, Lisbon, Portugal. ${ }^{2}$ Global Change Research Institute of the Czech Academy of Sciences, Bělidla 986/4a, CZ-60300 Brno, Czech Republic. ${ }^{3}$ CREAF, 08193 Cerdanyola del Vallès, Catalonia, Spain. ${ }^{4} \mathrm{CSIC}$, Global Ecology Unit CREAF-CSIC-UAB, 08193 Bellaterra, Catalonia, Spain. ${ }^{5}$ Servei de Ressonàcia Magnètica Nuclear, Universitat Autònoma de Barcelona, 08193 Cerdanyola del Vallès, Catalonia, Spain. ${ }^{6}$ Centre for Ecology, Evolution and Environmental Changes (CE3C), Faculdade de Ciências, Universidade de Lisboa, Lisbon, Portugal. ${ }^{7}$ Linking Landscape, Environment, Agriculture and Food (LEAF), Instituto Superior de Agronomia (ISA), Universidade de Lisboa, Lisbon, Portugal. ${ }^{8}$ Laboratório de FTICR e Espectrometria de Massa Estrutural, Departamento de Química e Bioquímica, Faculdade de Ciências, Universidade de Lisboa, Lisbon, Portugal. ${ }^{9} \mathrm{CEAUL}$ - Centro de Estatística e Aplicações, Faculdade de Ciências, Universidade de Lisboa, Lisbon, Portugal. ${ }^{10}$ Spanish National Research Council, Madrid, Spain. ${ }^{凶}$ email: mgsebastiana@fc.ul.pt
} 
a better performance of ECM plants against abiotic and biotic stress, such as drought, soil salinity, heavy metal contamination and protection from pathogen $\mathrm{s}^{2-5}$. Therefore, there is an increasing interest in using mycorrhizas to enhance the resilience of plants to the increasingly stressful environmental conditions and to aid in the desired reduction of fungicide and fertilizer application. Thus, studies on the molecular basis of mycorrhization are fundamental to be able to use the advantages of this plant symbiotic interactions, either by simple inoculation or by more complex approaches such as genome editing to copy mycorrhizal benefits to crop plants.

Cork oak (Quercus suber) is an evergreen tree species found in the western part of the Mediterranean basin where it is exploited for the production of cork, the thick outer bark layer that covers the trunk and can be extracted periodically without any harm to the tree, which regenerates back a new cork layer that can be peeled again from the tree. This sustainable agro-forestry system, with low human intervention is characterized by a high biodiversity of both plant and animal species (https://forest.jrc.ec.europa.eu/en/european-atlas/). However, cork oak forests are threatened by fire, agricultural expansion, diseases and climate change. Therefore, the plantation of new areas with cork oak is mandatory and could benefit from the use of mycorrhizal seedlings to increase survival of new plantations in the hot and dry regions of the western Mediterranean region.

This study aims to provide a comprehensive view of the metabolic alterations occurring in roots upon ECM symbiosis establishment using the cork oak - Pisolithus tinctorius ECM interaction as a model. Both partners efficiently form ECMs in greenhouse conditions, and have been the subject of transcriptomics and proteomics studies by our team, that have revealed some important molecular aspects of the regulation and metabolism of their symbiotic relationship ${ }^{6,7}$.

Currently, metabolomics technologies allow the separation and identification of a wide range of metabolites providing a global overview of the qualitative and quantitative changes of plant primary and secondary metabolites $^{8-11}$. However, few studies have addressed metabolic alterations underlying ECM symbiosis ${ }^{2,12-14}$. Previous untargeted metabolomics studies have shown a strong effect of ECM symbiosis on primary and secondary poplar root metabolism, mainly on aromatic acid, organic acid and fatty acids which were induced, while sugars were reduced ${ }^{12}$. On the other hand, investigation of the metabolic alterations in roots of an oak tree species colonized by the ECM fungus Tuber indicum indicated the accumulation of carbohydrates and organic acids ${ }^{13}$. Studies on the pre-symbiotic phase of ECM interaction in Eucalyptus grandis roots have shown a symbiotic specific plant metabolic response associated to the suppression of plant metabolism, which can be crucial in determining the outcome of the interaction ${ }^{14}$.

Using two untargeted metabolomics technologies, Proton Nuclear Magnetic Resonance $\left({ }^{1} \mathrm{H}\right.$ NMR) spectroscopy and Direct Infusion Fourier-Transform Ion Cyclotron Resonance Mass Spectrometry (DI-FT-ICR-MS), we analysed the metabolome of ECM roots. We detected major metabolic alterations associated with ECM formation in the roots of cork oak, and some of them were investigated in deeper detail by gene expression analysis. The impact of the identified changes is discussed in relation to the plant-fungus ECM symbiotic program.

\section{Results}

Effect of $P$. tinctorius on cork oak root colonization and growth. The ergosterol concentration was significantly higher in $P$. tinctorius inoculated roots relative to the non-inoculated plants, indicating the formation of a functional ECM symbiosis in cork oak roots upon inoculation (Fig. 1a). It is known that ECM symbiosis can have a positive effect on the growth and nutrient acquisition of colonized plants ${ }^{1}$. P. tinctorius inoculation had a positive effect on root growth, significantly increasing root biomass $(P<0.05)$, with mycorrhizal plants showing 1.89 times more root fresh weight than non-inoculated plants (Fig. 1b). Leaf biomass and root $\mathrm{N}$ concentration were not significantly affected by the P. tinctorius inoculation (Supplementary Table S1). Microscopic observations of the inoculated roots confirmed the ECM colonization of the cork oak roots by P. tinctorius. Typical ECM structures, such as the fungal mantle and the Hartig net were clearly observed in roots, 8 weeks after $P$. tinctorius inoculation (Fig. 1c,d). The level of $P$. tinctorius biomass (estimated by the concentration of ergosterol) in mycorrhizal roots was found to be $7 \%$ (Supplementary Table S1).

P. tinctorius colonization induces major metabolic changes in cork oak roots. NMR analysis of mycorrhizal and non-inoculated roots enabled the identification of 15 different metabolites (Supplementary Table S2 and Supplementary Fig. S1). A principal component analysis (PCA) of the different metabolites as variables and the different treatments as cases, showed a clear separation between mycorrhizal and non-inoculated roots suggesting a different metabolic response (Fig. 2a). Inoculation with $P$. tinctorius had a strong effect on several individual peaks that presented significant differences between mycorrhizal and non-inoculated roots $(P<0.05)$ (Figs. 2b, 3). Mycorrhizal roots showed higher levels of the amino acid's GABA and alanine, the sugar $\beta$-glucose, the organic acid citrate and the aromatic unknown compound 1 . In contrast, the organic osmolytes quercitol (a polyol) and glycine-betaine, the sugars $\alpha$-glucose and fructose, and the organic acids malate and lactate, showed decreased levels in mycorrhizal roots.

The FT-ICR analysis of the two metabolite fractions of mycorrhizal and non-inoculated roots revealed a mean number of about 4400 peaks per replicate in ESI + mode and 400 in ESI - ionization modes (Supplementary Table S3). The van Krevelen diagrams representing elemental formulas of mycorrhizal and non-inoculated roots revealed the high chemical diversity present in those samples (Supplementary Fig. S2). Notable differences between treatments were detected, with mycorrhizal roots showing fewer elemental formulas, specifically in areas corresponding to polyketides and carbohydrates. PCA analysis showed clear metabolic differences between mycorrhizal and non-inoculated roots (Fig. 4a), with the first two PCs explaining $64.7 \%$ of the total variance of the data, suggesting that both PC's are sufficient to discriminate between the inoculation treatments (mycorrhizal and non-inoculated). To access which of the metabolites are most responsible for the observed differences that correlated with $P$. tinctorius inoculation, we performed a supervised analysis using PLS-DA and used VIP 


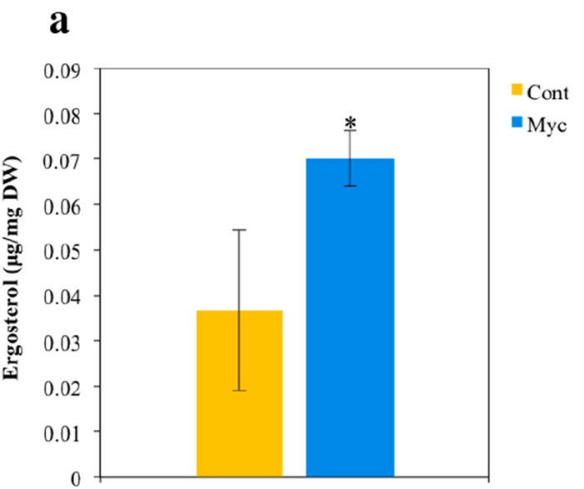

\section{b}
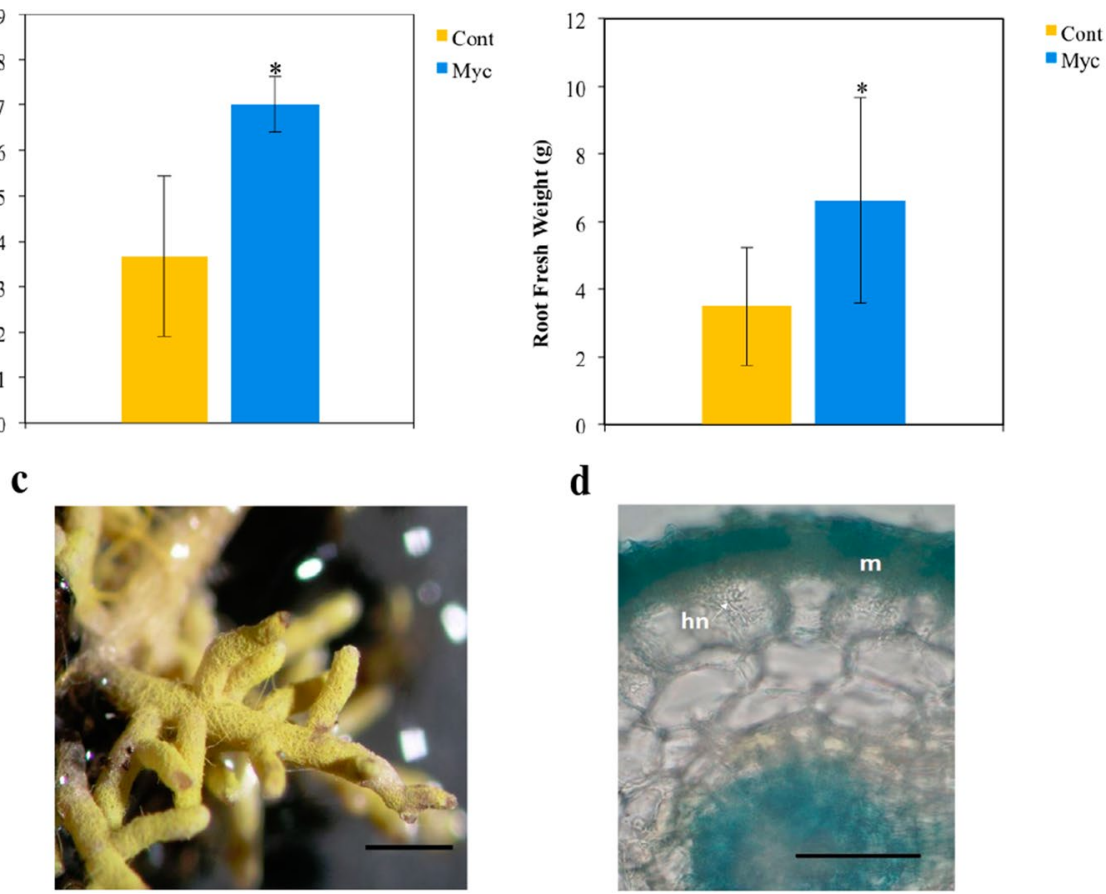

d

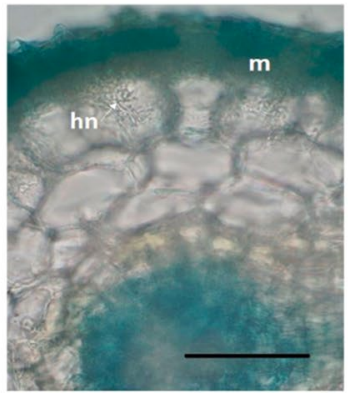

Figure 1. Fungal root colonization and plant growth of mycorrhizal (Myc) and non-inoculated (Cont) cork oak plants, 8 weeks after $P$. tinctorius inoculation. (a) Ergosterol root content. Data represent the mean of 3 independent biological replicates (each composed of a pool of 3 individual root systems) \pm SD (b) Root fresh weight. Data represent the mean of 15 independent plants \pm SD. ${ }^{\star}$ Indicates statistical differences at the level $P<0.05$ [t-test for (a) and Man-Whitney $U$ test for (b)]. (c) Colonized root showing the hyphal mantle. Scale bar $=1 \mathrm{~mm}$. (d) Representative trypan blue staining of a colonized root showing the fungal mantle (m) and the hartig net (hn). Scale bar $=50 \mu \mathrm{m}$.

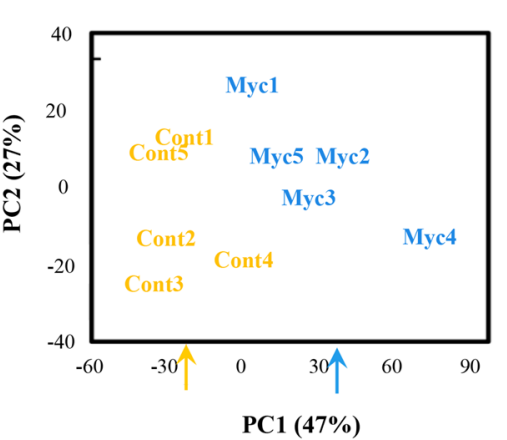

b

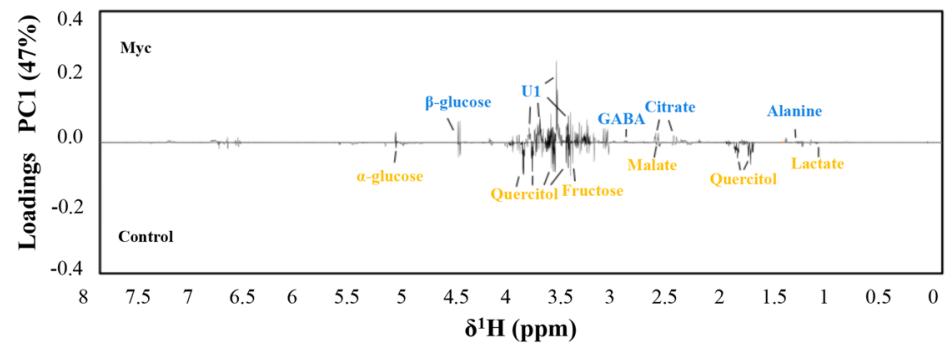

Figure 2. Overview of the metabolic reprograming (analysed by $1 \mathrm{H} \mathrm{NMR}$ ) in mycorrhizal (Myc) and noninoculated (Cont.) roots interpreted using PCA. (a) Biplot of the second principal component (PC2) versus the first principal component (PC1) scores. (b) Loading values of the first principal component (PC1). The molecular name of the elucidated peaks with significantly different concentration in mycorrhizal compared to non-inoculated roots is highlighted; blue corresponds to metabolites with higher concentrations in mycorrhizal roots and orange corresponds to those with higher concentration in non-inoculated roots.

scores to establish the importance of mass features. The scores plot showed a clear separation between mycorrhizal and non-inoculated groups (Fig. 4b), suggesting a separated root metabolite profile corresponding to each treatment. Internal cross-validation showed a value of $\mathrm{Q}^{2}$ above 0.8 , indicative of a good predictive performance of the PLS-DA model, with a maximum $\mathrm{Q}^{2}$ achieved with 3 PLS components (Fig. 4c). Masses with the highest discriminative capacity (VIP score $>1$ ) were selected as the most important metabolites that contributed for the separation of the metabolic changes identified. The VIP score describes a quantitative estimation of the discriminatory power of each individual feature ${ }^{15}$ and VIP $>1$ is commonly used as a cut-off for selecting the most 


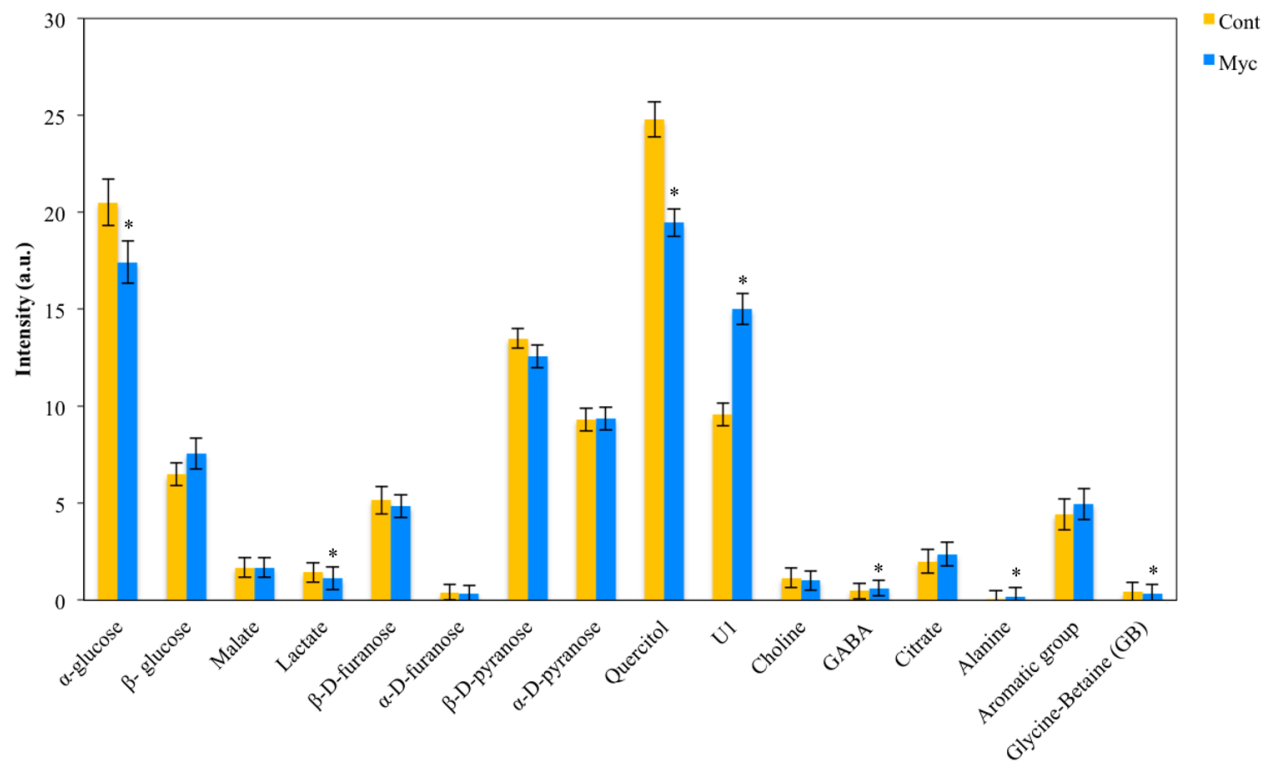

Figure 3. Comparisons between mycorrhizal (Myc) and non-inoculated (Cont) peak areas of root metabolites identified by NMR analysis. ${ }^{\star}$ Indicates statistical differences at the level $P<0.05$ (t-test).

important features separating the defined classes of samples in PLS-DA ${ }^{14,16,17}$. Using the MassTrix interface we were able to putatively annotate 61 metabolites discriminating between the inoculation treatments, including 37 with decreased levels, and 24 with increased levels in mycorrhizal roots compared to non-inoculated controls (Table 1; Supplementary Table S4). Classification of these metabolites into chemical classes revealed that inoculation with $P$. tinctorius affected 6 major classes of compounds: phytochemical compounds, lipids, carbohydrates, amino acids, nucleic acids and vitamins (Fig. 5). Mycorrhizal colonization had a strong effect on root primary metabolism, mainly on metabolites putatively corresponding to lipids and carbohydrates, including many putative fatty acids and monosaccharides, but also affected secondary metabolism, altering some phytochemical compounds, such as putative alkaloids, terpenoids and oxylipin-related compounds (Table 1; Supplementary Table S4). Among the differentially accumulated metabolites involved in primary metabolism, just like in the NMR analysis, several putative carbohydrates showed decreased levels in mycorrhizal roots, including isomers of glucose $(180.06392 \mathrm{Da})$ and sucrose $(342.11615 \mathrm{Da})$, and also of other carbohydrates related to the biosynthesis of sugar alcohols (polyols), such as sorbitol (sorbitol 6-phosphate, 262.04583 Da) and manosylglycerate (2-( $\alpha$-Dmannosyl)-3-phosphoglycerate, 348.04570 Da) (Fig. 6; Table 1; Supplementary Table S4).

Unlike what was found using NMR, which revealed increased levels of certain amino acids in mycorrhizal roots, metabolites putatively involved in amino acid metabolism detected by FT-ICR were found to be mostly less abundant in mycorrhizal roots, including compounds putatively involved in tyrosine metabolism (3-(3,4-dihydroxyphenyl)pyruvate) and histidine metabolism (imidazole-4-acetaldehyde) (Table 1; Supplementary Table S4).

Also, a decrease in the levels of putative fatty acids was detected in the mycorrhizal roots, including decreases in isomers of dodecanoic acid (200.17793 Da), hexadecanoic acid (256.23960 Da), hydroxydocosanoic acid $(356.32887 \mathrm{Da})$, hexadecanedioic acid (286.21400 Da), tricosanedioic acid (384.32363 Da) and oxo-tetradecanoic acid (242.18749 Da) (Fig. 6; Table 1; Supplementary Table S4). Other putative lipophilic compounds also less accumulated in the mycorrhizal roots, include putative monoacylglycerols, such as MG(18:0/0:0/0:0), MG(0:0/16:0/0:0) and MG(0:0/14:0/0:0) (Table 1; Supplementary Table S4). Regarding metabolites in the secondary metabolism category, a significant decrease in putative phenolic compounds which are known to be involved in the biosynthesis of hydrolysable tannins, such as 1-O-galloyl-beta-D-glucose (332.07468 Da) and ellagic acid $(302.00659 \mathrm{Da})$ was detected in mycorrhizal roots (Fig. 6; Table 1; Supplementary Table S4). Other secondary compounds matched metabolites related to plant stress response mechanisms, such as those putatively involved in the oxylipin pathway. In this context we identified decreased levels of a compound putatively corresponding to tuberonic acid glucoside $(388.17218 \mathrm{Da})$ in mycorrhizal roots, while the levels of two compounds putatively corresponding to 9-oxononanoic acid (172.11006 Da) and traumatic acid (228.13565 Da) were increased (Fig. 6; Table 1; Supplementary Table S4). In addition, metabolites in the terpenoids category, showed increased levels in colonized roots, including putative terpene glycosides [e.g. bryophyllin A (472.20845 Da); rhodexin A $(558.28118 \mathrm{Da})]$ and compounds putatively involved in the biosynthesis of terpenoids and steroids [e.g. (S)2,3-epoxysqualene (410.41212 Da)] (Fig. 6; Table 1; Supplementary Table S4). Other compounds related to defense, such as putative alkaloids, were identified in high numbers in cork oak roots, some being up- but most being down-accumulated in the P. tinctorius mycorrhizal roots (Table 1; Supplementary Table S4). Another identified compound implicated in the interaction of plants with microorganisms, which was increased in the mycorrhizal cork oak roots was a metabolite putatively corresponding to pyridoxamine (vitamin B6) (168.09029 Da) (Fig. 6; Table 1; Supplementary Table S4). 


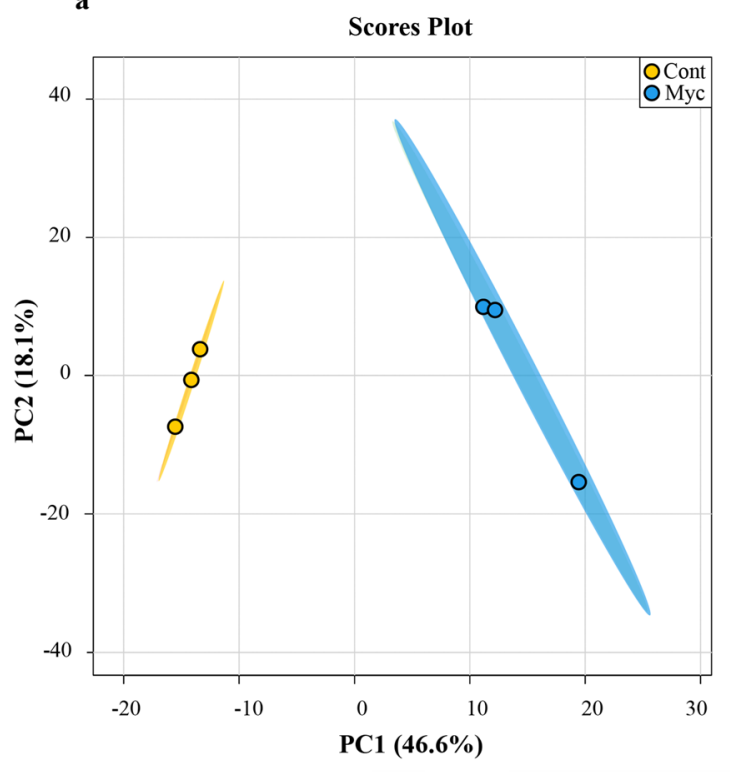

b

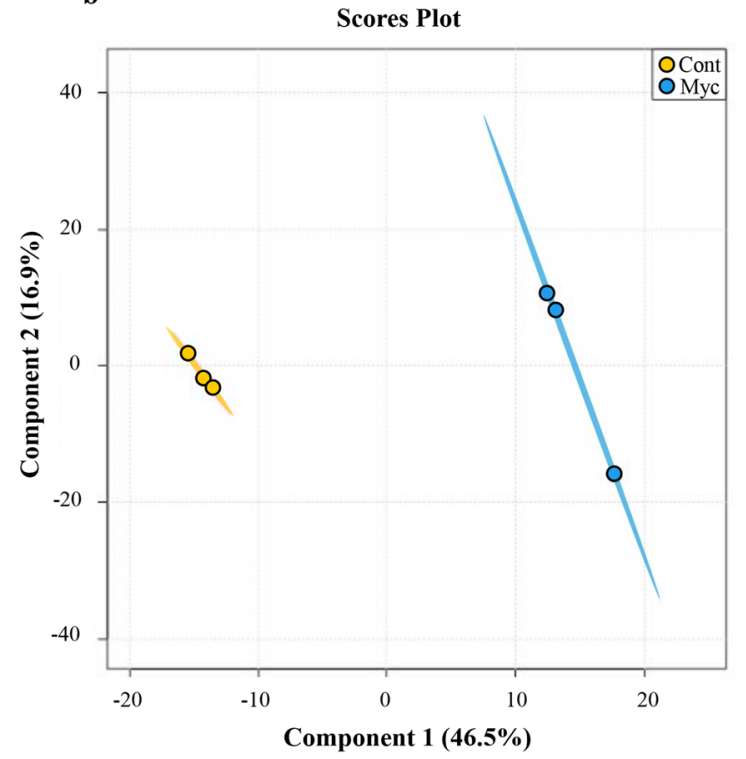

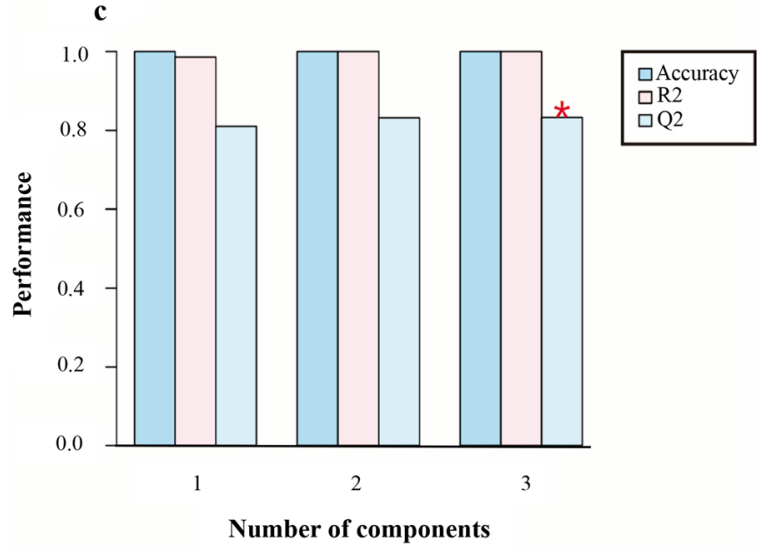

Figure 4. Overview of the metabolic reprograming analysed by FT-ICR in mycorrhizal and non-inoculated roots interpreted using PCA and PLS-DA. (a) PCA scores plot of mycorrhizal (Myc) and non-inoculated (Cont) cork oak roots. Each point represents a biological replicate (b) PLS-DA scores plot of mycorrhizal (Myc) and non-inoculated (Cont) cork oak roots. Each point represents a biological replicate. (c) Cross-validation scores plot of the PLS-DA for the classification into mycorrhizal and non-inoculated groups using the FT-ICR data as a function of the number of PLS components. The number of components that maximizes Q2 is indicated with a star. Charts were produced with the free available on-line software MetaboAnalyst v4.0 (https://www.metab oanalyst.ca/).

Evaluation of the expression of genes involved on GABA biosynthesis in mycorrhizal roots. Since our metabolomic analysis pointed to the involvement of GABA in ECM symbiosis and since recognition of pathogens and symbionts has proven to trigger GABA accumulation in several plant species ${ }^{18}$, we decided to investigate in more detail the molecular basis of the increased levels of GABA found in mycorrhizal roots. Results from the qPCR analysis (Fig. 7) show that the cork oak GAD1 transcript encoding the root specific $\mathrm{GAD}^{19}$ was increased in mycorrhizal roots relative to non-inoculated roots, while the expression of GABAT, which degrades GABA into succinic acid semialdehyde, was decreased. The accumulation of GABA seems to be of plant origin since transcription of the P. tinctorius GAD gene was barely detected in our qPCR analysis. The 2 genes encoding polyamine oxidases (PAO2 and PAO4) were increased in mycorrhizal roots, showing that GABA accumulation in cork oak mycorrhizal roots could result from polyamine catabolism (Fig. 7).

\section{Discussion}

In our experiment, the level of ergosterol concentration of mycorrhizal roots is consistent with a highly compatible interaction between cork oak and the P. tinctorius fungal strain used in our analysis. Increases in root biomass, as detected in cork oak mycorrhizal roots in the present study, are a common response during the interaction of tree roots with ECM fungi ${ }^{20}$. Enhanced root surface is most likely beneficial to the plant and fungus, increasing the potential for the uptake of fungal-derived nutrients by the plant and of plant-derived carbon by the fungus. 


\begin{tabular}{|c|c|c|c|c|c|}
\hline Neutral mass (Da) & Putative compound identity & Major class & Secondary class & VIP Score* & $\log 2(\mathrm{FC})$ myc vs cont \\
\hline 436.25858 & LPA $(0: 0 / 18: 1(9 \mathrm{Z}))$ & Lipids & Glycerophospholipids & 1.044 & -2.426 \\
\hline 424.29647 & LPA (18:0e/0:0) & Lipids & Glycerophospholipids & 1.024 & 2.100 \\
\hline 246.05016 & Glycerophosphoglycerol & Lipids & Glycerophospholipids & 1.143 & 2.998 \\
\hline 369.32455 & $\begin{array}{l}\text { N-stearoyl GABA\#N-palmitoyl } \\
\text { isoleucine\#N-palmitoyl leucine }\end{array}$ & Lipids & Fatty amides & 1.007 & -2.169 \\
\hline 341.29252 & Stearoylglycine\#N-palmitoyl GABA & Lipids & Fatty amides & 1.086 & 2.402 \\
\hline 283.28694 & Octadecanamide & Lipids & Fatty amides & 1.038 & -2.225 \\
\hline 383.34046 & $\mathrm{~N}$-stearoyl valine & Lipids & Fatty amides & 1.004 & 2.534 \\
\hline 281.27209 & Oleamide & Lipids & Fatty amides & 1.070 & -2.589 \\
\hline 248.17698 & 16:4(4Z,7Z,10Z,13Z) & Lipids & Fatty acids & 1.469 & 2.729 \\
\hline 340.33514 & Docosanoic acid & Lipids & Fatty acids & 1.121 & 2.742 \\
\hline 270.25520 & $\begin{array}{l}\text { Palmitic acid methyl ester\#Margaric } \\
\text { acid\#(+)-14-methyl palmitic acid\#15- } \\
\text { methyl palmitic acid }\end{array}$ & Lipids & Fatty acids & 1.092 & 2.454 \\
\hline 284.27080 & $\begin{array}{l}\text { Octadecanoic acid (Stearic } \\
\text { acid)\#(+)-Isostearic acid }\end{array}$ & Lipids & Fatty acids & 1.047 & 2.229 \\
\hline 200.17793 & Dodecanoic acid & Lipids & Fatty acids & 1.016 & -2.174 \\
\hline 298.28656 & $\begin{array}{l}\text { Nonadecanoic acid\#16-methyl-octadeca- } \\
\text { noic acid\#17-methyl-octadecanoic acid\#11- } \\
\text { methyl-octadecanoic acid }\end{array}$ & Lipids & Fatty acids & 1.023 & -2.121 \\
\hline 256.23960 & $\begin{array}{l}\text { Hexadecanoic acid (palmitic } \\
\text { acid)\#Isopalmitic acid\#13-methyl-penta- } \\
\text { decanoic acid }\end{array}$ & Lipids & Fatty acids & 1.006 & -2.138 \\
\hline 356.32887 & $\begin{array}{l}\text { 13-Hydroxydocosanoic acid } \# 2 \text {-Hydroxydo- } \\
\text { cosanoic acid } \# 22 \text {-Hydroxydocosanoic acid }\end{array}$ & Lipids & Fatty acids & 1.058 & -2.386 \\
\hline 384.32363 & Tricosanedioic acid & Lipids & Fatty acids & 1.449 & -2.549 \\
\hline 286.21400 & Hexadecanedioic acid & Lipids & Fatty acids & 1.019 & -2.193 \\
\hline 242.18749 & $\begin{array}{l}\text { 3-oxo-tetradecanoic acid\#6-oxo-tetrade- } \\
\text { canoic acid }\end{array}$ & Lipids & Fatty acids & 1.040 & -2.396 \\
\hline 128.05880 & $\begin{array}{l}\text { gamma-Amino-gamma- } \\
\text { cyanobutanoate\#alpha-Amino-gamma- } \\
\text { cyanobutanoate }\end{array}$ & Lipids & Fatty acids & 1.007 & -2.133 \\
\hline 358.30764 & MG(18:0/0:0/0:0)\#MG(0:0/18:0/0:0) & Lipids & Monoacylglycerols & 1.764 & -4.584 \\
\hline 358.30797 & MG(0:0/18:0/0:0)\# MG(18:0/0:0/0:0) & Lipids & Monoacylglycerols & 1.403 & -2.454 \\
\hline 330.27638 & MG(0:0/16:0/0:0)\#MG(16:0/0:0/0:0) & Lipids & Monoacylglycerols & 1.012 & -2.050 \\
\hline 302.24490 & MG(0:0/14:0/0:0)\#MG(14:0/0:0/0:0) & Lipids & Monoacylglycerols & 1.129 & -1.435 \\
\hline 250.11981 & Methylripariochromene A\#Ubiquinone Q1 & Lipids & Prenol lipids & 1.032 & -2.330 \\
\hline 347.07876 & Hydroxysanguinarine & Phytochemical compounds & Alkaloids & 1.549 & 3.145 \\
\hline 354.23015 & Aspidospermine & Phytochemical compounds & Alkaloids & 1.519 & -2.863 \\
\hline 315.14751 & $\begin{array}{l}\text { 3'-Hydroxy-N-methyl-(S)- } \\
\text { coclaurine\#(R)-Norreticuline\#(S)-Nor- } \\
\text { reticuline\#Cephalotaxine }\end{array}$ & Phytochemical compounds & Alkaloids & 1.459 & -2.540 \\
\hline 305.16269 & $\begin{array}{l}\text { (6S)-Hydroxyhyoscyamine\#Porritoxin\#Lun } \\
\text { acridine\#Anisodamine\#Convolamine }\end{array}$ & Phytochemical compounds & Alkaloids & 1.129 & 2.960 \\
\hline 240.14751 & Slaframine & Phytochemical compounds & Alkaloids & 1.054 & 2.165 \\
\hline 398.22030 & Desacetoxyvindoline\#Mitragynine & Phytochemical compounds & Alkaloids & 1.008 & -2.177 \\
\hline 385.15301 & Polycarpine & Phytochemical compounds & & 1.010 & 2.028 \\
\hline 430.21168 & Cinegalline & Phytochemical compounds & Alkaloids & 1.089 & -2.675 \\
\hline 287.24570 & Prosopinine & Lipids & Alkaloids & 1.040 & -2.240 \\
\hline 267.13732 & Ergine & Phytochemical compounds & Alkaloids & 1.007 & -2.028 \\
\hline 332.07468 & 1-O-Galloyl-beta-D-glucose & Phytochemical compounds & Phenolic compounds & 1.201 & -4.247 \\
\hline 302.00659 & Ellagic acid & Phytochemical compounds & Phenolic compounds & 1.099 & -1.782 \\
\hline 310.15629 & Dihydrocordoin & Phytochemical compounds & Flavonoids & 1.066 & 2.362 \\
\hline
\end{tabular}




\begin{tabular}{|c|c|c|c|c|c|}
\hline Neutral mass (Da) & Putative compound identity & Major class & Secondary class & VIP Score* & $\log 2(\mathrm{FC})$ myc vs cont \\
\hline 298.08401 & $\begin{array}{l}\text { Apigenin 7,4'-dimethyl ether\#Afror- } \\
\text { mosin\#Sayanedine\#Sayanedine\#Cladr } \\
\text { in\#Afrormosin\#Alfalone\#8-O-M } \\
\text { ethylretusin\#5-O-Methylbiocha- } \\
\text { nin A\#Pterocarpin\#Kuhlmannin\#4'- } \\
\text { Hydroxy-5,7-dimethoxy-4- } \\
\text { phenylcoumarin\#Tithonine\#7-Hydroxy- } \\
\text { 3',4'-dimethoxyflavone\#5-Hydroxy- } \\
\text { 6,2'-dimethoxyflavone\#5-Hydroxy- } \\
\text { 7,2'-dimethoxyflavone\#Syzalterin\#8- } \\
\text { Demethylsideroxylin\#Apigenin } \\
\text { 7,4'-dimethyl ether\#Baicalein } \\
\text { 5,6-dimethyl ether\#Mosloflavone\#7- } \\
\text { Hydroxy-5,8-Dimethoxyflavone\#7- } \\
\text { O-Methylwogonin\#5,7-Dihydroxy- } \\
\text { 3-methoxy-8-methylflavone\#3,5-Dihy- } \\
\text { droxy-7-methoxy-8-methylflavone\#3,5,7- } \\
\text { Trihydroxy-6,8-dimethylflavone\#3,7- } \\
\text { Dimethoxy-5-hydroxyflavone\#Galangin } \\
\text { 5,7-dimethyl ether\#Isoneobavachalcone\#N } \\
\text { eobavachalcone\#Lawinal }\end{array}$ & Phytochemical compounds & Flavonoids & 1.012 & 2.034 \\
\hline 228.13565 & Traumatic acid & Phytochemical compounds & Oxylipins & 1.008 & 2.019 \\
\hline 172.11006 & 9-Oxononanoic acid & Phytochemical compounds & Oxylipins & 1.414 & 2.422 \\
\hline 388.17218 & Tuberonic acid glucoside & Phytochemical compounds & Oxylipins & 1.015 & -2.071 \\
\hline 208.04069 & $\begin{array}{l}\text { 2-(2'-Methylthio)ethylmalic acid\#3-(2'- } \\
\text { Methylthio)ethylmalic acid }\end{array}$ & Phytochemical compounds & Glucosinolates & 1.489 & 2.826 \\
\hline 339.10701 & Vulgaxanthin-I & Phytochemical compounds & Amino acid related compounds & 1.012 & -2.052 \\
\hline 180.06392 & $\begin{array}{l}\text { D-Glucose\#alpha-D-Glucose\#beta-D- } \\
\text { Glucose\#beta-D-Glucoside\#D-Fructose\#L- } \\
\text { Fructose\#beta-D-Fructose\#Galactose\#D- } \\
\text { Galactose\#L-Galactose\#alpha-D- } \\
\text { Galactose\#myo-Inositol\#scyllo- } \\
\text { Inositol\#D-Mannose\#L-Sorbose\#D- } \\
\text { Sorbose\#D-Tagatose\#D-Allose\#L- } \\
\text { Fuconate\#L-Rhamnonate\#D-Altrose\#D- } \\
\text { Gulose\#D-Idose\#D-Talose\#D-Psicose\#L- } \\
\text {-Gulose\#Scyllitol }\end{array}$ & Carbohydrates & Monosaccharides & 1.375 & -2.362 \\
\hline 262.04583 & Sorbitol 6-phosphate & Carbohydrates & & 1.298 & -2.344 \\
\hline 307.09057 & S-Succinyldihydrolipoamide-E & Carbohydrates & & 1.015 & 2.568 \\
\hline 290.03955 & Sedoheptulose 7-phosphate & Carbohydrates & & 1.039 & 1.346 \\
\hline 348.04570 & 2-(alpha-D-Mannosyl)-3-phosphoglycerate & Carbohydrates & & 1.034 & -2.198 \\
\hline 342.11615 & $\begin{array}{l}\text { Sucrose\#Cellobiose\#Maltose\#Lactose\#Isom } \\
\text { altose\#Trehalose\#Galactinol\#Nigerose\#Ma } \\
\text { nnobiose\#Palatinose\#Laminaribiose\#Melib } \\
\text { iose\#Turanose }\end{array}$ & Carbohydrates & Oligosaccharides & 1.012 & -2.053 \\
\hline 260.03017 & $\begin{array}{l}\text { D-Fructose 6-phosphate\#D-Fructose } \\
\text { 1-phosphate\#beta-D-Fructose } \\
\text { 2-phosphate\#beta-D-Fructose } \\
\text { 6-phosphate\#D-Glucose 6-phosphate\#D- } \\
\text { Glucose 1-phosphate\#beta-D- } \\
\text { Glucose 1-phosphate\#alpha-D- } \\
\text { Glucose 6-phosphate\#beta-D-Glucose } \\
\text { 6-phosphate\#D-Mannose 6-phosphate\#D- } \\
\text { Mannose 1-phosphate\#D-Galactose } \\
\text { 6-phosphate\#alpha-D-Galactose } \\
\text { 1-phosphate\#D-Galactose 1-phosphate\#L- } \\
\text { Galactose 1-phosphate\#L-Galactose } \\
\text { 1-phosphate\#Inositol 1-phosphate\#myo- } \\
\text { Inositol 4-phosphate\#1D-myo- } \\
\text { Inositol 3-phosphate\#D-Myo-inositol } \\
\text { 4-phosphate\#D-arabino-3-Hexulose } \\
\text { 6-phosphate\#L-Gulose1-phosphate\#Dolichyl } \\
\text { phosphate D-mannose\#D-Tagatose } \\
\text { 6-phosphate\#Sorbose 1-phosphate\#D- } \\
\text { Allose 6-phosphate }\end{array}$ & Carbohydrates & & 1.010 & -1.679 \\
\hline 196.03657 & 3-(3,4-Dihydroxyphenyl) pyruvate & Amino acids & & 1.061 & -2.413 \\
\hline
\end{tabular}




\begin{tabular}{|l|l|l|l|l|l|}
\hline Neutral mass (Da) & Putative compound identity & Major class & Secondary class & VIP Score & Log2 (FC) $\mathbf{m y c}$ vs cont \\
\hline 308.04092 & $\begin{array}{l}\text { Uridine monophosphate\#Pseudouridine } \\
\text { 5'-phosphate\#Uridine } \\
\text { 3'-monophosphate\#Orotidine }\end{array}$ & Nucleic acids & Nucleotides & 1.040 & -2.335 \\
\hline 114.04325 & Deoxyuridine monophosphate & Nucleic acids & Nucleotides & 1.018 & -2.088 \\
\hline 168.09029 & Pyridoxamine & Vitamins and Cofactors & Vitamins & 1.005 & 2.004 \\
\hline
\end{tabular}

Table 1. Putative identities of the metabolites discriminating mycorrhizal (myc) roots from non-inoculated (cont) roots analyzed by FT-ICR. *VIP $>1$ indicates important features discriminating Myc from Cont treatments.

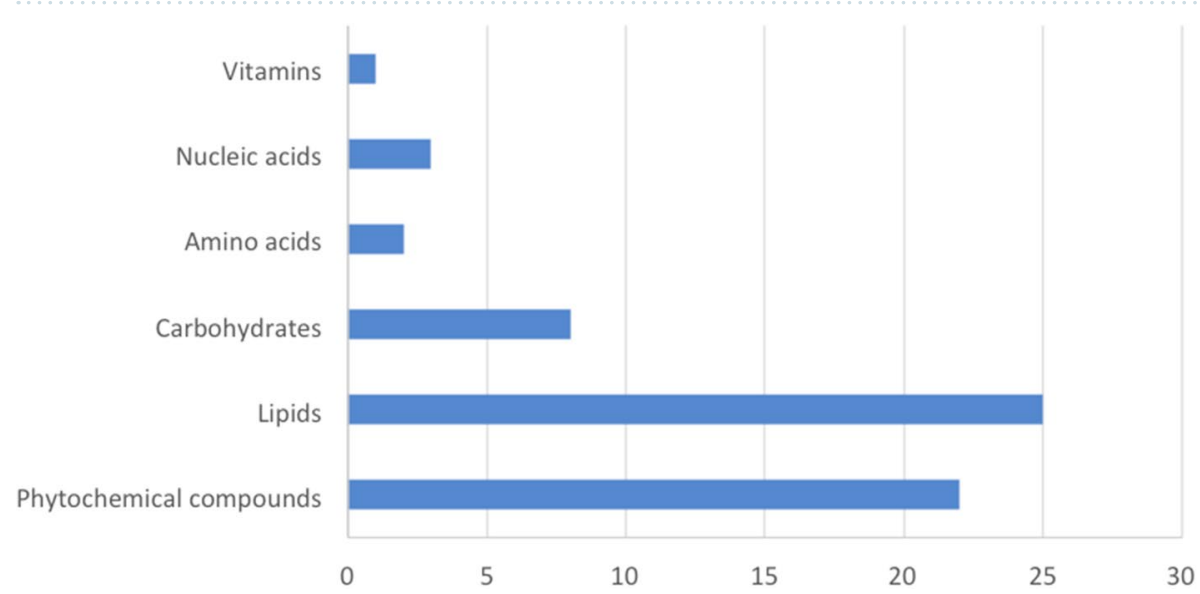

Figure 5. Chemical classes associated with the annotated metabolites discriminating mycorrhizal roots from non-inoculated roots (VIP $>1$ ) analysed by FT-ICR. The $\mathrm{x}$ axis corresponds to the number of annotated masses assigned to each class.

Our untargeted metabolic analysis confirmed that the metabolome of mycorrhizal oak roots is significantly different from that of non-inoculated roots. Since in our experiment the mycorrhizal roots had only an estimated $7 \%$ of $P$. tinctorius biomass we assumed that alterations are most likely occurring in the plant tissues. However, additional experimental verification would be needed to unequivocally prove the origin of the identified metabolites, such as prior isotopic labelling of $P$. tinctorius tissues followed by tracing of labelled fungal metabolites in the mycorrhizal roots ${ }^{14}$. Nevertheless, since most metabolites detected in the cork oak roots were decreased by P. tinctorius interaction, they are most likely plant metabolites.

We found that symbiosis with $P$. tinctorius decreased the levels of many metabolites in the mycorrhizal cork oak roots, in agreement with metabolomics studies by Wong et al. ${ }^{14}$, which showed a general repression of metabolites at the pre-mycorrhizal phase of the interaction of Eucalyptus grandis and Pisolithus microcarpus. More suppressed metabolites were associated with $P$. microcarpus strains with high root colonization potential, suggesting that decreased metabolic responses during the pre-symbiotic early stages could be crucial for successful ECM colonization at latter stages ${ }^{14}$. These results might suggest a common strategy of Pisolithus species to colonize the roots of their host plants.

Our results show that $P$. tinctorius was negatively associated with the level of several primary metabolites in the cork oak mycorrhizal roots, such as putative carbohydrates and fatty acids, which content was reduced. Likewise, secondary compounds putatively related to hydrolysable tannins were also reduced following $P$. tinctorius mycorrhization. In contrast, putative non-proteogenic amino acids, like GABA, and some putative secondary metabolites, such as terpenoids and oxylipins, had increased levels in mycorrhizal roots. Previous work on other ECM biological systems have also showed an extensive metabolic reprograming induced by mycorrhiza formation, affecting primary and secondary metabolism ${ }^{12,13}$, derived from the alterations at the transcript and protein levels, detected in several studies ${ }^{21}$.

It must be mentioned that the experiment present here, using young seedlings, does not fully express the endogenous rhythmic growth of oak tress which alternates between shoot and root flushes, that are related to parallel shifts in above- and below-ground allocation of carbon compounds ${ }^{22}$. Since we collected roots during the root growth period of oak seedlings (May-June) ${ }^{23}$, our experimental system might only have a parallel in the root flush period of older seedlings ${ }^{23}$, when carbon allocation is shifted to the roots ${ }^{24}$. The use of oak microcuttings would enable to surpass this limitation and examine the metabolic responses during the whole growth cycle, but these are only available for Quercus robur ${ }^{25}$.

Our NMR and FT-ICR analysis showed a reduction in the levels of putative compounds related to sugar and organic acid metabolism in mycorrhizal roots. Besides compounds putatively corresponding to sucrose and glucose, other putative carbohydrates involved in the biosynthesis of sugar alcohols (polyols), like quercitol and sorbitol, were less abundant in mycorrhizal roots when compared to non-inoculated controls. We also detected 


\section{Carbohydrates}
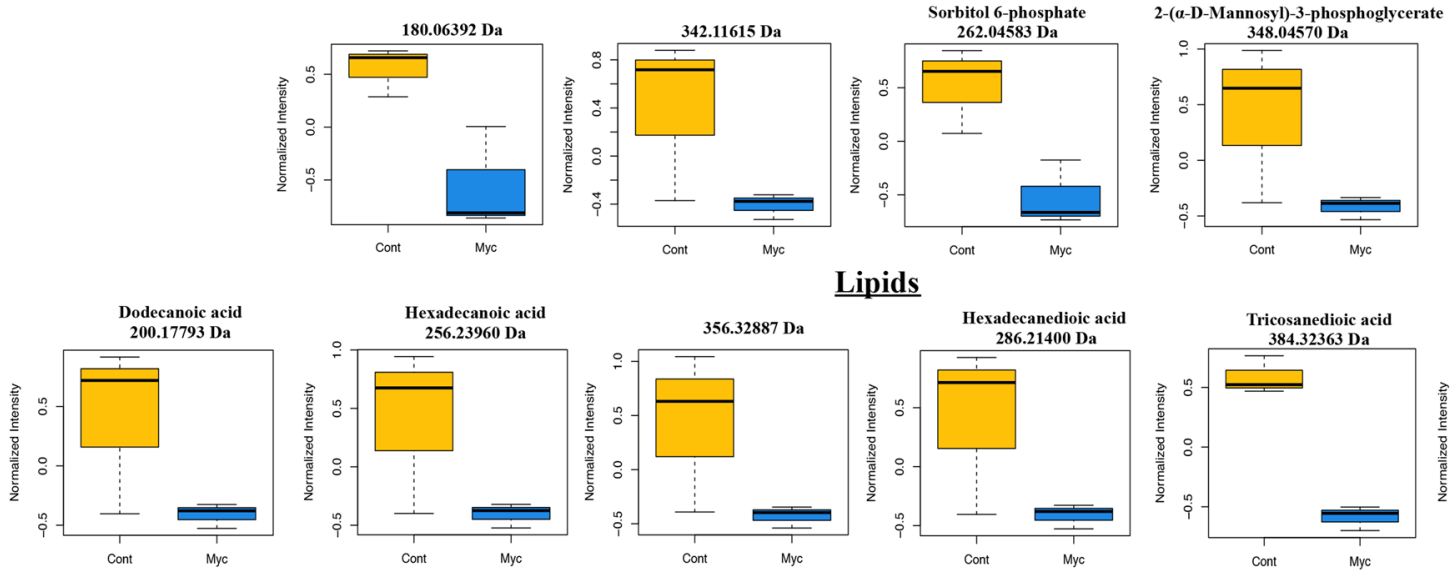

Lipids
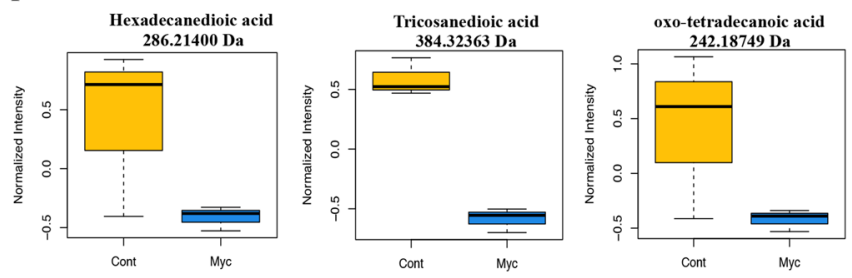

\section{Phenolic compounds}
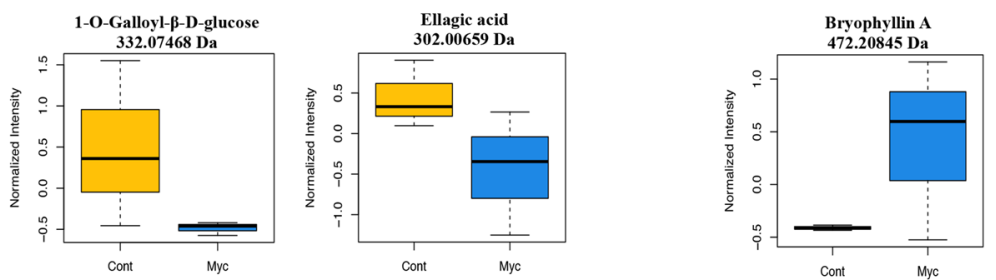

\section{Terpenoids}
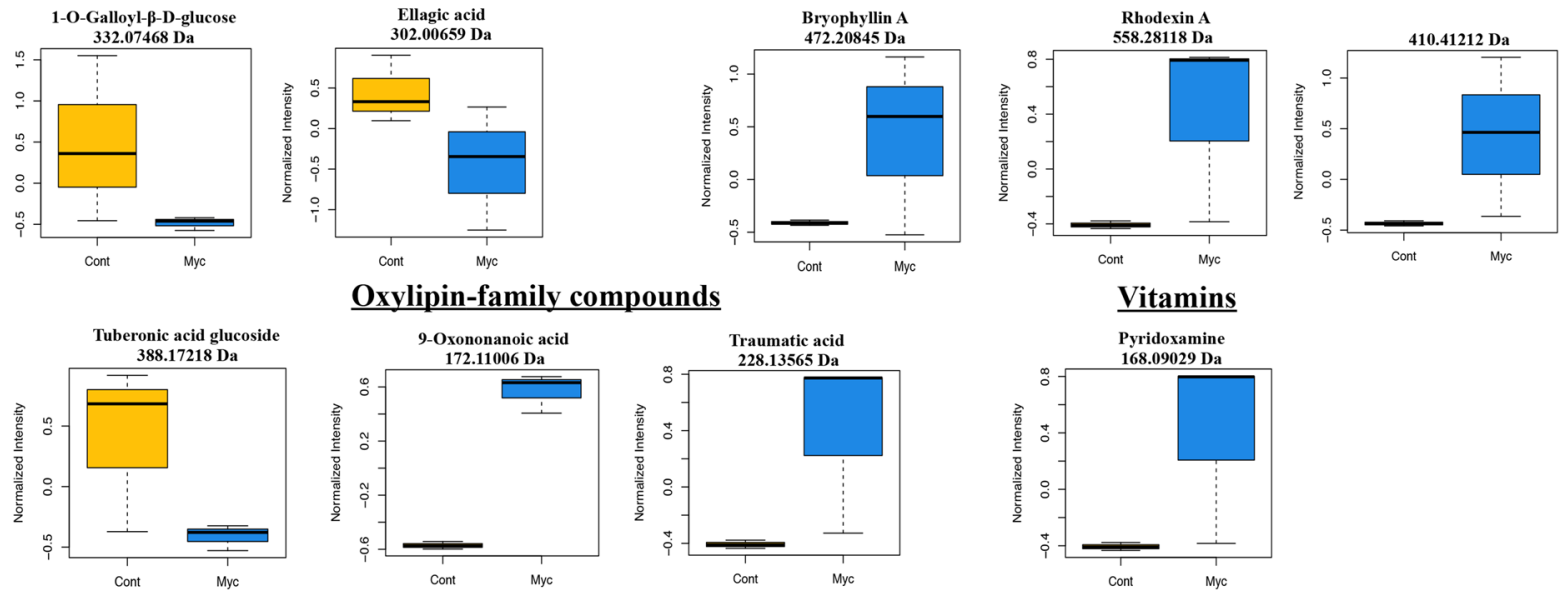

Figure 6. Accumulation profile of selected metabolites (VIP $>1$ ) in cork oak mycorrhizal roots. P. tinctorius inoculated roots $(\mathrm{Myc})$ and non-inoculated roots (Cont) were used for relative quantification by FT-ICR analysis. Compounds only tentatively identified are identified by an $\mathrm{m} / \mathrm{z}$ ratio. Charts were produced with the free available on-line software RStudio v1.2.5042 ${ }^{83}$.

lower levels of some organic acids, such as lactate and malate, and of the amino acid glycine-betaine. All these compounds have been identified in other studies as components of plant root exudates or associated to apoplastic protective barriers such as the bark tissues of tress ${ }^{26-28}$. Plants exude a considerable proportion (20-40\%) of their assimilates ${ }^{29}$, including sugars, amino acids, and organic acids which are believed to be passively lost from the root and used by rhizosphere-dwelling microbes ${ }^{30}$. It has long been acknowledged that carbon compounds in root exudates contribute to attract symbionts in the rhizosphere, like mycorrhizas and beneficial bacteria, that depend on this carbon supply for their survival ${ }^{29,31,32}$. ECM fungi, like $P$. tinctorius associate to the apoplast of roots where these compounds are most certainly exuded. Their decreased levels in the mycorrhizal roots, detected in our experiment, might indicate its transference to the fungus. Decreases in carbohydrates and organic acids were also detected in other ECM interactions ${ }^{12}$ and in previous studies on the Q. suber - P. tinctorius root symbiotic system ${ }^{7}$. Several of the identified compounds, such as those putatively corresponding to quercitol and glycine-betaine, are very abundant metabolites in tree species naturally subjected to hot and dry climate conditions, such as Q. suber, functioning as protective compatible solutes and osmoprotectors ${ }^{33,34}$. Therefore, their high availability in the roots from this species can be suggested since a growing body of evidence shows that carbon allocation to root exudates is driven by disposal of surplus carbon, exudates containing more of the elements that plants have in surplus ${ }^{29}$. According to Buscot and Herrmann ${ }^{35}$, ECM symbiosis is affected by the endogenous rhythmic growth of oaks, being stimulated during growth flushes that increase C resource availability in the roots (root flush). The positive effect in root biomass we observed in our experimental system is probably related to the stimulation of $P$. tinctorius symbiosis during root flush when plant $C$ resources allocated to the roots can be exchanged by fungal derived mineral nutrients, improving plant growth ${ }^{36}$. On the other hand, the decrease in carbohydrate levels detected in the mycorrhizal cork oak roots (relative to non-inoculated roots) is consistent with a sink effect exerted by $P$. tinctorius in order to assimilate part of the $\mathrm{C}$ resources accumulating in the roots during root flush. However, under our experimental conditions we cannot confirm that the identified $\mathrm{C}$ metabolites are being exuded by roots or used by $P$. tinctorius. On way to address this issue would be by isotopic tracing using those metabolites, combined with transcriptomic analysis to show that pathways leading to those compounds are not being repressed by the symbiotic interaction. 


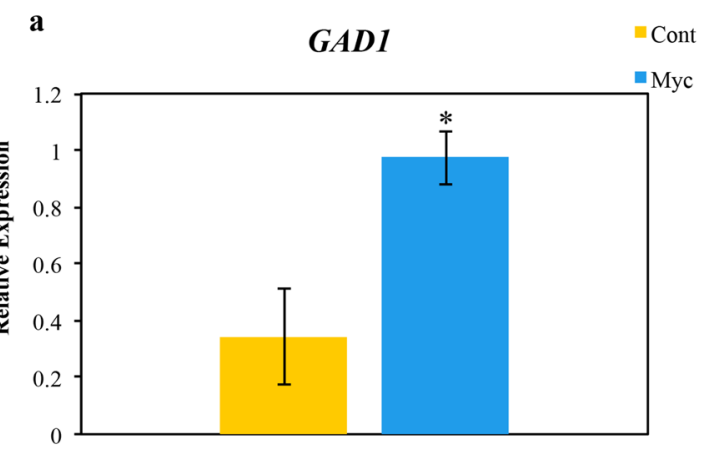

b

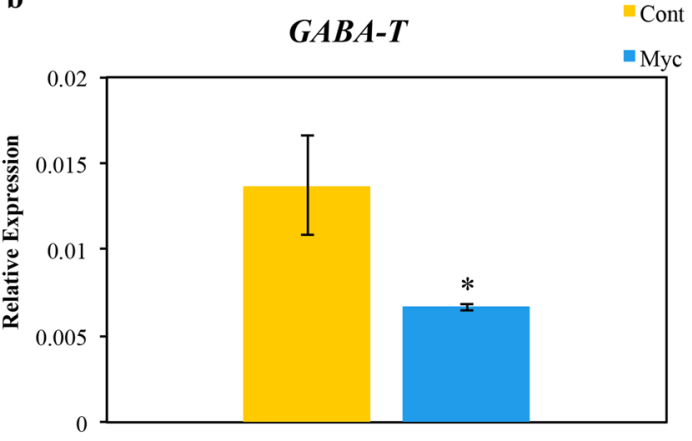

c

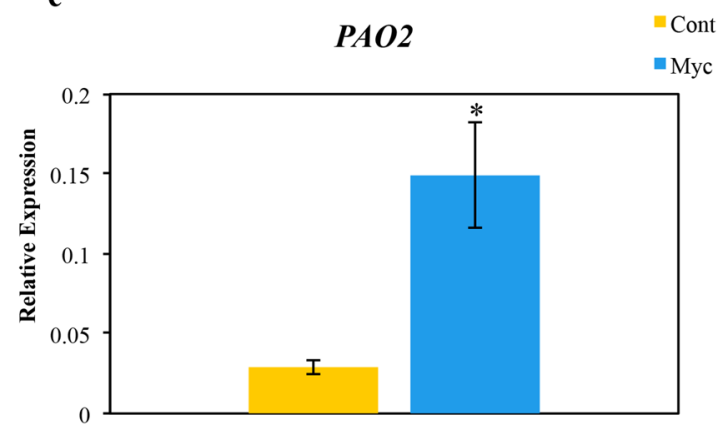

d

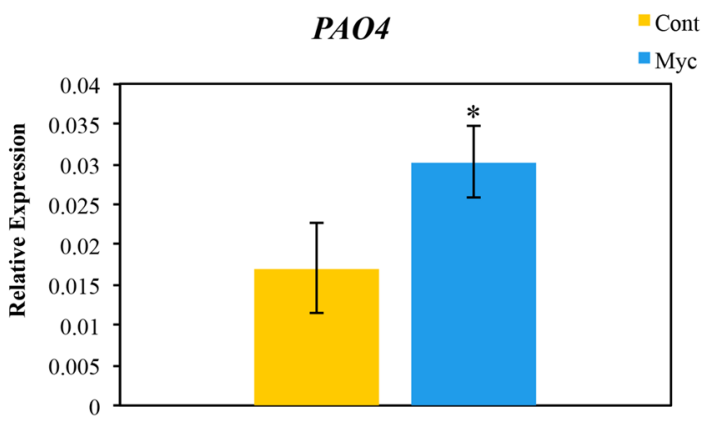

Figure 7. Genes expression profile of genes involved in GABA metabolism (GAD1, GABAT, PAO2, PAO4) analysed by real-time PCR. Bars represent averages \pm SD of 3 biological replicates of mycorrhizal (Myc) and noninoculated (Cont) cork oak plants. ${ }^{\star}$ Indicate statistical differences at the level $P<0.05$ (Mann-Whitney $U$ test).

One notable target of the metabolic reprograming induced by $P$. tinctorius in cork oak roots was at the level of lipid metabolism. A decrease in putative monoacylglycerols and fatty acids was observed in the mycorrhizal roots. Our results contrast with those reported in arbuscular mycorrhizas (AM), where the plant activates lipid biosynthesis, transferring fatty acids to the symbiotic fungus ${ }^{37}$. AM fungi are obligate biotrophs that depend on the plant partner not only for carbon supply but also for lipids, with several important genes needed to synthesize fatty acids being absent from the AM fungi genome ${ }^{38}$. However, ECM fungi have a more flexible lifestyle, living in association with plant host roots but surviving also in decaying soil organic matter. Studies have shown that ECM

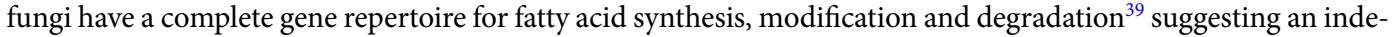
pendence from the plant host lipid metabolism. Most of the putative fatty acids detected as down-accumulated in the cork oak mycorrhizal roots, such as isomers of nonadecanoic acid, hexadecanoic acid, hydroxydocosanoic acid, tricosanedioic acid and hexadecanedioic acid are long-chain saturated fatty acids. Remarkably, these class of fatty acids, together with monoacylglycerols (which were also decreased in our experiment), are major components of the suberin and cutin of bark, a layer that accumulates in the outermost face of shoots and roots of trees, protecting tissues from desiccation and pathogen attack ${ }^{27,40,41}$. Other compounds detected in lower levels in the cork oak ECM roots, such as those putatively corresponding to tannins, are also common components of suberin and bark tissues ${ }^{27,41}$. These results, pointing to a decrease in metabolites putatively involved in root protective bark layers are in line with the alterations in plant gene expression consistent with a cell wall loosening process that have been reported in several studies on $\mathrm{ECMs}^{6,42}$. This might contribute to facilitate fungal progression in the plant apoplast and symbiosis establishment.

Alterations in compounds putatively involved in the oxylipin pathway were detected in the mycorrhizal roots in our experimental system. Compounds in this pathway, such as jasmonates (JA), are associated to plant defense against pathogens. Increases in oxylipins, such as traumatic acid and azelaic acid, were also detected in ECM roots of Quercus aliena with Tuber indicum ${ }^{13}$, which may indicate a common response of oaks to ECM fungi. Other compounds putatively involved in response to biotic and abiotic stress detected in the mycorrhizal cork oak roots, matched putative compounds in the terpenoid and flavonoid category. Studies have shown that plant colonization by symbiotic organism such as ECM fungi enhances the production of flavonoids and terpenoids in the host roots ${ }^{14}$, mycorrhizal plants having generally reduced attacks by root-feeding insects ${ }^{43}$. In this context, a recent metabolomics study by Almeida et al. ${ }^{44}$ identified flavonoids and terpenoids as metabolites involved in the ability of cork oak to cope with drought stress, which suggests a common metabolic response to symbiosis and abiotic stress, also detected in other studies ${ }^{2}$. ECM cork oak roots showed increased levels of a compound putatively corresponding to pyridoxamine, a vitamin B6 pathway compound described to participate in mycorrhizal-induced abiotic stress tolerance in tomato plants ${ }^{45}$, and in mycorrhiza induced resistance (MIR) against pathogens ${ }^{46}$.

Increasing evidence support a role of GABA in protecting plants against major abiotic and biotic stressors ${ }^{18}$. Previous studies using RNAseq suggest an increase in GABA synthesis on ECM roots of cork oak with $P$. 
tinctorius $^{6}$. Therefore, to investigate in deeper detail the role of GABA increases detected in our ECM system we analysed the expression of cork oak genes involved in the GABA shunt, the major pathway leading to GABA synthesis and catabolism in plants ${ }^{18}$. The increased levels of glutamate decarboxylase (GAD1) transcription in mycorrhizal roots, while GABA transaminase (GABAT) expression was reduced, shows that reactions leading to GABA production are being activated, while those associated to GABA catabolism are impaired. The expression of polyamine oxidases (PAO), which can be oxidized to GABA ${ }^{18}$ was increased in the P. tinctorius mycorrhizal cork oak roots most likely also contributing to GABA synthesis in this tissue. Studies have found that increases in GABA during pathogenic interactions in plants can be derived from fungi ${ }^{47,48}$. However, this is unlikely in our experiment, since $P$. tinctorius GAD transcription was barely detected in mycorrhizal roots. Though important gaps of knowledge remain to be filled in this sense, there are several reports suggesting the involvement of GABA and related compounds in other symbiotic interactions ${ }^{49}$ including AM interactions, such as the increased GABA levels and GAD activity in mycorrhizal tomato roots ${ }^{50-52}$, or the recent discovery of a Pisolithus albus effector which enhances the biosynthesis of polyamines, favouring the colonization of the host Eucalyptus grandis ${ }^{53}$. However, to validate the role played by GABA in the ECM symbiosis between cork oak and P. tinctorius, additional experimental verification would be needed, such as treatment of cork oak plants with GABA or GABA homologs, followed by evaluation of plant tolerance to abiotic and/or biotic stress. Overall, these results constitute an indication that ECM fungi induce the activation of defense responses, probably to control ECM fungal growth within limits, which might contribute to the priming of defences that protects mycorrhizal plants from pathogenic microorganisms and plant pests ${ }^{4}$. Induction of plant defences by various types of mutualistic organisms including ECM fungi is a common event associated to the recognition of an alien organism ${ }^{2}$. However, even in low compatibility symbiotic interactions (negative effect on plant growth) the fungal partner is recognized as symbiont, and no mechanisms characteristic of the negative pathogenic/parasitic interactions, such as ROS burst or increased lignification, are activated ${ }^{54}$.

Defense responses in mycorrhizal oaks seem to be affected by resource allocation related to the rhythmic growth patterns of the tree, since during the shoot flush the increased sink strength due to dual inoculation (with a nematode and an ECM fungus) results in a suppression of plant defenses, likely a response of the plant to reallocate reserves aboveground ${ }^{55}$. Since we collected roots during root flush, when carbon resources are allocated to roots, a negative effect on plant defense responses related to the sink effect exerted by $P$. tinctorius is not expected, which is in accordance with our results showing the accumulation of compounds putatively involved in plant defense.

In conclusion, using a non-targeted metabolomics approach we were able to characterize the metabolic reprogramming occurring in cork oak roots following ECM formation with $P$. tinctorius. A shift in primary and secondary root metabolism was observed. Several of the metabolic changes detected are putatively related to plant defense and immunity mechanisms and could be involved in the mycorrhizal enhancement of plant stress resistance that has been reported in several studies. In this context, compounds such as GABA, pyridoxamine, or terpenoids have been previously shown to prime plant immunity against pathogen attack, improving also resistance to abiotic stresses in symbiotic plants ${ }^{45,49}$. These are interesting targets for a detailed functional study aiming to investigate their role in ECMs and the associated molecular mechanisms related to improved resistance against biotic and abiotic stresses.

\section{Methods}

Plant material and $P$. tinctorius inoculation. The $P$. tinctorius strain Pt 23 was maintained in pure culture on BAF agar medium containing glucose $1 \%$, in the darkness at $23{ }^{\circ} \mathrm{C}$. Quercus suber acorns were surface sterilized with a $10 \%$ commercial bleach solution and germinated in trays containing soil acquired from a gardening store $(80-150 \mathrm{mg} / \mathrm{L} \mathrm{N}, 80-150 \mathrm{mg} / \mathrm{L} \mathrm{P} 2 \mathrm{O} 5,300-500 \mathrm{mg} / \mathrm{L} \mathrm{K2O}, \mathrm{pH}(\mathrm{CaCl} 2)$ 5.5-6.5, organic matter $>70 \%)$. Soil was autoclaved $\left(121^{\circ}, 1 \mathrm{~atm} ., 60 \mathrm{~min}\right)$ before use. Four-week old plantlets were transferred to 2L pots containing autoclaved soil and inoculated with $P$. tinctorius inoculum according to Sebastiana et al. ${ }^{7}$. Control plants were treated with an autoclaved $P$. tinctorius inoculum. Plants were maintained in soil in a greenhouse under a randomized block design. A total of 15 plants were used for each treatment. Eight weeks upon P. tinctorius inoculation, mycorrhizal and non-inoculated plants were harvested for analysis (May-June). First, the root system of each plant was gently washed with demineralized water. The fresh weight of leaves and roots (whole root system) was determined. Significant differences between mycorrhizal and non-inoculated plants were assessed using T-test $(P$ value $\leq 0.05)$, after testing data for normality using the Shapiro Wilk test and for equality of variance using Levene's test. An aliquot of 3 individual inoculated and non-inoculated root systems was reserved for mycorrhizal root colonization assessment. Next, lateral roots showing at least one mycorrhiza were cut at the intersection with the principal root with a scalpel blade and immediately frozen in liquid nitrogen for further analysis. In parallel, lateral roots from non-inoculated plants were collected in the same way. At collection, roots from mycorrhizal and non-inoculated plants were visually inspected for the presence of fungal mycelium, besides that of $P$. tinctorius. No signs of mycelium from other fungi were detected. Roots from 3 independent plants were pooled together constituting in one biological replicate. In total, 5-3 biological replicates from mycorrhizal roots and non-inoculated roots were collected for analysis. Each root biological replicate was grounded to a fine powder using liquid nitrogen and stored at $-80^{\circ} \mathrm{C}$.

Root ergosterol concentration and fungal biomass. Root ergosterol concentration was determined in roots using 3 biological replicates, each composed of a pool of 3 individual root systems, and in P. tinctorius pure culture, as described in Sebastiana et al. ${ }^{7}$. Significant differences between $P$. tinctorius inoculated and noninoculated plants were assessed using Mann-Whitney $U$ test $(P$ value $\leq 0.05)$. The $\%$ of $P$. tinctorius biomass in mycorrhizal roots was estimated by calculating the ergosterol in mycorrhizal roots (ergosterol in mycorrhizal 
roots - ergosterol in non-inoculated roots) relative to the ergosterol of a P. tinctorius pure culture (100 percentage ergosterol) (Supplementary Table S1).

Nitrogen root concentration. The nitrogen concentration of roots was determined at the Stable Isotopes and Instrumental Analysis Facility, Faculty of Sciences, Lisbon University. Three biological replicates, each consisting of a pool of roots from 3 plants, were analysed for each treatment. Frozen root material was dried at $70^{\circ}$ $\mathrm{C}$ for $72 \mathrm{~h}$ and grounded in a mill (Retsch, Germany) to a homogenous fine powder for isotopic analysis. After grinding, samples were used for $\mathrm{N}$ percentage calculation, according to Rodrigues et al. ${ }^{56}$, on a EuroEA 3000 CHNS-O Elemental Analyzer (EuroVector, Milan), with a TDC detector. Root N concentration was defined as $\%$ of dry weight. Significant differences between $P$. tinctorius inoculated and non-inoculated plants were assessed using Mann-Whitney $\mathrm{U}$ test $(P$ value $\leq 0 \cdot 05)$.

${ }^{1} \mathrm{H}$ NMR analysis. $\quad{ }^{1} \mathrm{H}$ NMR sample preparation. Freshly collected root samples were immediately packed and frozen in liquid nitrogen. This frozen root material (5 biological replicates from mycorrhizal and noninoculated roots) was lyophilized, its metabolites were extracted using a mixture of water/methanol and then transferred into NMR sample tubes, as described in Rivas-Ubach et al. ${ }^{57}$. Trimethylsilyl propionic acid (TSP), was used as internal standard.

${ }^{1} \mathrm{H}$ NMR fingerprinting. High-resolution ${ }^{1} \mathrm{H}$ NMR spectroscopy measurements were conducted using a Bruker AVANCE 600 spectrometer equipped with an automatic sample changer and a multinuclear triple resonance (TBI) probe (all from Bruker, Rheinstetten, Germany) at a field strength of $14.1 \mathrm{~T}\left(600.13 \mathrm{MHz}{ }^{1} \mathrm{H}\right.$ frequency), following the methodology described in Rivas-Ubach et al. ${ }^{57}$ and Gargallo-Garriga et al. ${ }^{58}$. The determination process was controlled using TopSpin 2.1 software (Bruker, Rheinstetten, Germany).

${ }^{1} H$ NMR data analysis. A bucketing process and peak mean centering and standardization for statistical analyses of the 1D ${ }^{1} \mathrm{H}-\mathrm{NMR}$ spectra were conducted using AMIX software (Bruker, Rheinstetten, Germany), as described in Rivas-Ubach et al. ${ }^{57}$. Multivariate analyses (PCAs based on correlation matrices) were performed to detect patterns of sample ordination according with the metabolic profile. Differences in PCA scores between mycorrhizal and non-inoculated roots were tested by one-way ANOVA. Differences in individual peaks (integral values of each metabolite) between mycorrhizal and non-inoculated roots were determined using T-test. The normality of each variable was tested by Kolmogorov-Smirnov test. All variables followed normal distributions. Statistical analyses were performed by using vegan, mixOmics, nlme, ggplo2, ade4 and FactoMineR packages in R v3.5.1 Core software.

${ }^{1}$ H NMR metabolite identification. The sample extracts were also used for the acquisition of the 2D NMR sampling spectra. The metabolite identification was done as described in Rivas-Ubach et al. ${ }^{57}$ based on the structural elucidation of the spectra. Briefly, hydrogens (protons) connected through three to five chemical bonds were identified using $2 \mathrm{D}{ }^{1} \mathrm{H}-\mathrm{NMR}$ homonuclear COSY and TOCSY correlations, whereas ${ }^{1} \mathrm{H}-{ }^{1} \mathrm{H}$ NOESY method was used to determine connections between distinct parts of target molecules. Finally, heteronuclear ${ }^{1} \mathrm{H}-{ }^{13} \mathrm{C}$ HSQC and HMBC methods were used to identify the carbon skeleton of each target molecule. Spectra were referenced to TSP $\left({ }^{1} \mathrm{H}\right.$ and ${ }^{13} \mathrm{C}$ at $\left.\delta 0.00 \mathrm{ppm}\right)$. When possible, assignments were confirmed with described databases ${ }^{59-72}$.

FT-ICR analysis. FT-ICR sample preparation. Sample preparation and metabolite extraction were performed following the protocol previously described ${ }^{73}$. Briefly, frozen root powder $(100 \mathrm{mg})$ from each sample (3 biological replicates from mycorrhizal roots and non-inoculated roots, and $P$. tinctorius fungal mycelium growing in pure culture) was extracted using $1 \mathrm{ml}$ of solvent mixture (methanol, chloroform and water (2:2:1, v/v/v). Samples were then vortexed $(1 \mathrm{~min})$ and maintained for $15 \mathrm{~min}$ in an orbital shaker for metabolite extraction. Samples were centrifuged (1000 g, $15 \mathrm{~min}$, room temperature) for phase separation: chloroform fraction and aqueous-methanol fraction. The chloroform (C) fraction was collected, re-centrifuged and lyophilized at $-40{ }^{\circ} \mathrm{C}$. The aqueous-methanol fraction was subjected to solid-phase extraction (LiChrolut RP-18columns, Merck) and the metabolites were sequentially eluted (twice) with $1 \mathrm{~mL}$ of water and $1 \mathrm{ml}$ of methanol. The water (W) fraction was lyophilized at $-40{ }^{\circ} \mathrm{C}$, while the methanol $(\mathrm{M})$ fraction was evaporated under a stream of nitrogen. The $\mathrm{W}$ and $\mathrm{C}$ fractions were solubilised in a water/methanol solution $(1: 1, \mathrm{v} / \mathrm{v})$, whereas the $\mathrm{M}$ fraction was solubilised in the respective pure solvent.

FT-ICR mass spectra acquisition. Each sample was diluted 1000-fold in the appropriate solvent: M fraction was diluted in the same solvent for positive- $\left(\mathrm{ESI}^{+}\right)$and negative-ion $\left(\mathrm{ESI}^{-}\right)$mode analysis; $\mathrm{W}$ and $\mathrm{C}$ fractions were diluted in methanol for $\mathrm{ESI}^{+}$and in methanol/water (1:1) for $\mathrm{ESI}^{-}$. Leucine enkephalin (YGGFL, Sigma Aldrich) was added to all the fractions $(0.5 \mu \mathrm{g} / \mathrm{mL})$ and was used for internal calibration and for quality assessment of analytical precision $\left([\mathrm{M}+\mathrm{H}]^{+}=556.276575 \mathrm{Da}\right.$ or $\left.[\mathrm{M}-\mathrm{H}]^{-}=554.260925 \mathrm{Da}\right)$. The diluted extracts from each sample were analysed by direct infusion in an Apex Qe 7-T Fourier Transform Ion Cyclotron Resonance Mass Spectrometer (FT-ICR-MS, Bruker, Bremen, Germany). Spectra were acquired using electrospray ionization (ESI) in positive $\left(\mathrm{ESI}^{+}\right)$and negative $\left(\mathrm{ESI}^{-}\right)$modes, as described previously ${ }^{73}$. Mass spectra were recorded with an acquisition size of $512 \mathrm{k}$, in the 100 to $1000 \mathrm{~m} / \mathrm{z}$ mass range ${ }^{73}$.

FT-ICR data analysis. Mass spectra were analysed using the software package Data Analysis 5.0 (Bruker, Bremen, Germany). Single point calibration was performed with the leucine enkephalin standard, both in posi- 
tive and negative ionization modes. Mass peak lists were exported as ASCII files, with a signal-to-noise ratio of 4. Data Analysis 5.0 was also used to obtain putative assigned compound formulas. Smart formula tool was used following the upper formula: $\mathrm{C}_{78} \mathrm{H}_{126} \mathrm{O}_{27} \mathrm{P}_{9} \mathrm{~S}_{14} \mathrm{~N}_{20}$ and lower formula: $\mathrm{C}_{1} \mathrm{H}_{1} \mathrm{O}_{0} \mathrm{P}_{0} \mathrm{~S}_{0} \mathrm{~N}_{0}$ as described in Kind and Fiehn $^{74}$. Formulas were exported to plot $\mathrm{H} / \mathrm{C}$ ratios against $\mathrm{O} / \mathrm{C}$ ratios for mycorrhizal and non-inoculated roots (Van Krevelen diagrams). Peak lists of the different samples were aligned into a single matrix with a maximum error of $2.0 \mathrm{ppm}$ deviation between replicates using an in-house Python script, as previously described ${ }^{73}$. Only the ions detected in at least 2 biological replicates were considered. Features with 3 intensity values or 3 missing values in at least one of the treatments were selected for further analysis. The acquired data matrices were further analysed with the MetaboAnalyst (4.0) package $^{75}$ (https://www.metaboanalyst.ca/). Peak intensities were median normalized, log transformed, and Pareto scaled. A multivariate data analysis approach was performed using Partial Component Analysis (PCA) and Principal Least Square Discriminant Analysis (PLS-DA). A PCA was applied to examine global metabolite variation triggered by the two treatment groups: mycorrhizal inoculation and control. Then, a PLS-DA was used to classify the groups segregation and identify the most important metabolites explaining the changes in metabolic profiles between treatments. Metabolites with variable importance in projection values (VIP) $>1$ were selected as the most important features having the highest discriminating capacity between the treatments.

FT-ICR metabolite identification. The putative identification of metabolite identities was conducted by searching against chemical public databases, such as Kyoto Encyclopaedia of Genes and Genomes (KEGG), Human Metabolome Database (HMDB) and LIPID MAPS, using the MassTRIX interface ${ }^{76}$ (http://masstrix.org). The final mass list was uploaded to the MassTRIX 3 server with the following parameters: scan mode was either positive or negative; adducts considered were $\mathrm{M}+\mathrm{H}, \mathrm{M}+\mathrm{K}$ and $\mathrm{M}+\mathrm{Na}$ for $\mathrm{ESI}^{+}$data and $\mathrm{M}-\mathrm{H}$ and $\mathrm{M}+\mathrm{Cl}$ for $\mathrm{ESI}^{-}$data; maximum $\mathrm{m} / \mathrm{z}$ deviation was $3 \mathrm{ppm}$; search was performed in the "KEGG/hmdb/LIPID MAPS without isotopes" databases. Metabolite annotation was further accomplished using the annotation pipeline developed by Nascimento et al. ${ }^{77}$. Search results matching to synthetic, bacteria- or animal-derived compounds were discarded. For compounds with multiple annotations a manual curation was performed, and the putative metabolite classes were described based on the common chemical structure.

Analysis of gene expression by qPCR. The expression of genes involved in gamma-aminobutyric acid (GABA) synthesis and degradation was analysed by qPCR. In order to design cork oak specific primers, the following Arabidopsis protein sequences encoding GABA metabolic enzymes were used for searching the NCBI database (TBLASTN) restricted to Quercus suber (taxid: 58,331): GAD1 (AT5G17330.1), GABAT (AT3G22200.2), PAO4 (AT1G65840.1), and PAO2 (AT2G43020.1). A P. tinctorius GAD mRNA sequence annotated as glutamate decarboxylase (jgi|Pisti1|18,961|gm1.1382_g) was retrieved from the JGI database and used for specific primer design. Total RNA from mycorrhizal and non-inoculated roots was extracted using the protocol of Wan and Wilkins ${ }^{78}$. Total RNA was treated with DNase using the Turbo DNA-free kit (Invitrogen) and first-strand cDNA was synthesized from purified total RNA using Reverse Transcriptase (RevertAid H Minus M-MuLV Reverse Transcriptase, Fermentas) and Oligo dT primer. Three biological replicates (composed of roots from 3 independent plants each) were analysed per treatment. qPCR was performed as described previously ${ }^{79}$. Information on genes, primer sequences and qPCR amplification are shown in Supplementary Table S5. Melting curve analysis was used to identify non-specific PCR products (Supplementary Fig. S3). Relative quantification of specific transcript levels was calculated using the Livak and Schmittgen method ${ }^{80}$. Expression values were normalized using the cork oak elongation factor-1 $\alpha$ gene (EF-1 $\alpha$ ), previously reported as a reference gene in cork oak-P. tinctorius $\mathrm{ECMs}^{7}$. Significant differences between mycorrhizal and non-inoculated roots were assessed using Mann-Whitney $U$ test $(P$ value $\leq 0.05)$.

\section{Data availability}

The datasets generated during the current study are available in figshare data repository with the identifiers https://doi.org/10.6084/m9.figshare.13379852.v1 ${ }^{81}$ and https://doi.org/10.6084/m9.figshare.14096213.v1 ${ }^{82}$.

Received: 21 December 2020; Accepted: 6 April 2021

Published online: 21 April 2021

\section{References}

1. Smith, S. E. \& Read, D. 9 - Nitrogen mobilization and nutrition in ectomycorrhizal plants. In Mycorrhizal Symbiosis 3rd edn (eds Smith, S. E. \& Read, D.) 321-348 (Academic Press, Cambridge, 2008). https://doi.org/10.1016/B978-012370526-6.50010-6.

2. Luo, Z.-B. et al. Upgrading root physiology for stress tolerance by ectomycorrhizas: insights from metabolite and transcriptional profiling into reprogramming for stress anticipation. Plant Physiol. 151, 1902-1917 (2009).

3. Gianinazzi, S. et al. Agroecology: the key role of arbuscular mycorrhizas in ecosystem services. Mycorrhiza 20, 519-530 (2010).

4. Jung, S. C., Martinez-Medina, A., Lopez-Raez, J. A. \& Pozo, M. J. Mycorrhiza-induced resistance and priming of plant defenses. J. Chem. Ecol. 38, 651-664 (2012).

5. Song, Y., Chen, D., Lu, K., Sun, Z. \& Zeng, R. Enhanced tomato disease resistance primed by arbuscular mycorrhizal fungus. Front. Plant Sci. 6, 786 (2015).

6. Sebastiana, M. et al. Oak root response to ectomycorrhizal symbiosis establishment: RNA-seq derived transcript identification and expression profiling. PLoS ONE 9, e98376 (2014).

7. Sebastiana, M. et al. Oak protein profile alterations upon root colonization by an ectomycorrhizal fungus. Mycorrhiza 27, 109-128 (2017).

8. Gougeon, R. D. et al. The chemodiversity of wines can reveal a metabologeography expression of cooperage oak wood. Proc. Natl. Acad. Sci. 106, 9174-9179 (2009). 
9. Sardans, J., Peñuelas, J. \& Rivas-Ubach, A. Ecological metabolomics: overview of current developments and future challenges. Chemoecology 21, 191-225 (2011).

10. Rivero, J., Gamir, J., Aroca, R., Pozo, M. J. \& Flors, V. Metabolic transition in mycorrhizal tomato roots. Front. Microbiol. 6, 598 (2015).

11. Gargallo-Garriga, A. et al. Root exudate metabolomes change under drought and show limited capacity for recovery. Sci. Rep. 8, 12696 (2018).

12. Tschaplinski, T. J. et al. Populus trichocarpa and Populus deltoides exhibit different metabolomic responses to colonization by the symbiotic fungus Laccaria bicolor. Mol. Plant-Microbe Interact. 27, 546-556 (2014).

13. Li, Q. et al. Chinese black truffle (Tuber indicum) alters the ectomycorrhizosphere and endoectomycosphere microbiome and metabolic profiles of the host tree Quercus aliena. Front. Microbiol. 9, 2202 (2018).

14. Wong, J. W. H. et al. The influence of contrasting microbial lifestyles on the pre-symbiotic metabolite responses of Eucalyptus grandis roots. Front. Ecol. Evol. 7, 10 (2019).

15. Cho, H.-W. et al. Discovery of metabolite features for the modelling and analysis of high-resolution NMR spectra. Int. J. Data Min. Bioinform. 2, 176-192 (2008).

16. Weljie, A. M., Bondareva, A., Zang, P. \& Jirik, F. R. 1H NMR metabolomics identification of markers of hypoxia-induced metabolic shifts in a breast cancer model system. J. Biomol. NMR 49, 185-193 (2011).

17. Duft, R. G. et al. Metabolomics approach in the investigation of metabolic changes in obese men after 24 weeks of combined training. J. Proteome Res. 16, 2151-2159 (2017).

18. Tarkowski, ŁP., Signorelli, S. \& Höfte, M. $\gamma$-Aminobutyric acid and related amino acids in plant immune responses: emerging mechanisms of action. Plant. Cell Environ. 43, 1103-1116 (2020).

19. Bouché, N., Fait, A., Zik, M. \& Fromm, H. The root-specific glutamate decarboxylase (GAD1) is essential for sustaining GABA levels in Arabidopsis. Plant Mol. Biol. 55, 315-325 (2004).

20. Ditengou, F. A. et al. Volatile signalling by sesquiterpenes from ectomycorrhizal fungi reprogrammes root architecture. Nat. Commun. 6, 6279 (2015).

21. Mello, A. \& Balestrini, R. Recent insights on biological and ecological aspects of ectomycorrhizal fungi and their interactions. Front. Microbiol. 9, 216 (2018).

22. Herrmann, S. et al. Endogenous rhythmic growth in oak trees is regulated by internal clocks rather than resource availability. J. Exp. Bot. 66, 7113-7127 (2015).

23. Reich, P. B., Teskey, R. O., Johnson, P. S. \& Hinckley, T. M. Periodic root and shoot growth in oak. For. Sci. 26, 590-598 (1980).

24. Herrmann, S. et al. Endogenous rhythmic growth, a trait suitable for the study of interplays between multitrophic interactions and tree development. Perspect. Plant Ecol. Evol. Syst. 19, 40-48 (2016).

25. Herrmann, S., Munch, J.-C. \& Buscot, F. A gnotobiotic culture system with oak microcuttings to study specific effects of mycobionts on plant morphology before, and in the early phase of, ectomycorrhiza formation by Paxillus involutus and Piloderma croceum. New Phytol. 138, 203-212 (1998).

26. Badri, D. V. \& Vivanco, J. M. Regulation and function of root exudates. Plant Cell Environ. 32, 666-681 (2009).

27. Ucar, M. B. \& Ucar, G. Characterization of methanol extracts from Quercus hartwissiana wood and bark. Chem. Nat. Compd. 47, 697-703 (2011).

28. Escudero, N., Marhuenda-Egea, F. C., Ibanco-Cañete, R., Zavala-Gonzalez, E. A. \& Lopez-Llorca, L. V. A metabolomic approach to study the rhizodeposition in the tritrophic interaction: tomato Pochonia chlamydosporia and Meloidogyne javanica. Metabolomics 10, 788-804 (2014).

29. Prescott, C. E. et al. Surplus carbon drives allocation and plant-soil interactions. Trends Ecol. Evol. 35, 1110-1118 (2020).

30. Canarini, A., Kaiser, C., Merchant, A., Richter, A. \& Wanek, W. Root Exudation of primary metabolites: mechanisms and their roles in plant responses to environmental stimuli. Front. Plant Sci. 10, 157 (2019).

31. Rudrappa, T., Czymmek, K. J., Paré, P. W. \& Bais, H. P. Root-secreted malic acid recruits beneficial soil bacteria. Plant Physiol. 148, $1547-1556$ (2008).

32. van Dam, N. M. \& Bouwmeester, H. J. Metabolomics in the rhizosphere: tapping into belowground chemical communication. Trends Plant Sci. 21, 256-265 (2016).

33. Merchant, A., Tausz, M., Arndt, S. K. \& Adams, M. A. Cyclitols and carbohydrates in leaves and roots of 13 Eucalyptus species suggest contrasting physiological responses to water deficit. Plant Cell Environ. 29, 2017-2029 (2006).

34. Passarinho, J. A. P., Lamosa, P., Baeta, J. P., Santos, H. \& Ricardo, C. P. P. Annual changes in the concentration of minerals and organic compounds of Quercus suber leaves. Physiol. Plant. 127, 100-110 (2006).

35. Buscot, F. \& Herrmann, S. At the frontier between basiodiomycotes and plants: reciprocal interactions between mycorrhiza formation and root development in an in vitro system with oaks and hymenomycetes. In Frontiers in Basidiomycote Mycology (eds Agerer, R. et al.) 361-376 (IHW, Eching, 2004).

36. Smith, S. E. \& Read, D. 4 - Growth and carbon economy of arbuscular mycorrhizal symbionts. In Mycorrhizal Symbiosis 3rd edn (eds Smith, S. E. \& Read, D.) (Academic Press, Cambridge, 2008). https://doi.org/10.1016/B978-012370526-6.50010-6.

37. Keymer, A. et al. Lipid transfer from plants to arbuscular mycorrhiza fungi. Elife 6, e29107 (2017).

38. Tang, N. et al. A Survey of the gene repertoire of Gigaspora rosea unravels conserved features among Glomeromycota for obligate biotrophy. Front. Microbiol. 7, 233 (2016).

39. Reich, M. et al. Fatty acid metabolism in the ectomycorrhizal fungus Laccaria bicolor. New Phytol. 182, 950-964 (2009).

40. Olivella, M. À. \& Río, J. C. D. Suberin composition from different bark layers of Quercus suber L by Py-GC/MS in the presence of tetramethylammonium hydroxide (TMAH). BioResources 6, 4936-4941 (2011).

41. Ferreira, J. P. A., Miranda, I., Sousa, V. B. \& Pereira, H. Chemical composition of barks from Quercus faginea trees and characterization of their lipophilic and polar extracts. PLoS ONE 13, 1-18 (2018).

42. Balestrini, R. \& Bonfante, P. Cell wall remodeling in mycorrhizal symbiosis: a way towards biotrophism. Front. Plant Sci. 5, 237 (2014).

43. Sharma, E., Anand, G. \& Kapoor, R. Terpenoids in plant and arbuscular mycorrhiza-reinforced defence against herbivorous insects. Ann. Bot. 119, 791-801 (2017).

44. Almeida, T. et al. In-depth analysis of the Quercus suber metabolome under drought stress and recovery reveals potential key metabolic players. Plant Sci. 299, 110606 (2020).

45. Rivero, J., Âlvarez, D., Flors, V., Azcón-Aguilar, C. \& Pozo, M. J. Root metabolic plasticity underlies functional diversity in mycorrhiza-enhanced stress tolerance in tomato. New Phytol. 220, 1322-1336 (2018).

46. Sanchez-Bel, P. et al. The nitrogen availability interferes with mycorrhiza-induced resistance against Botrytis cinerea in tomato. Front. Microbiol. 7, 1598 (2016).

47. Copley, T. R., Duggavathi, R. \& Jabaji, S. The transcriptional landscape of Rhizoctonia solani AG1-IA during infection of soybean as defined by RNA-seq. PLoS ONE 12, e0184095 (2017).

48. Brilli, M. et al. A multi-omics study of the grapevine-downy mildew (Plasmopara viticola) pathosystem unveils a complex protein coding- and noncoding-based arms race during infection. Sci. Rep. 8, 757 (2018).

49. Hua, M.D.-S. et al. Metabolomic compounds identified in Piriformospora indica-colonized Chinese cabbage roots delineate symbiotic functions of the interaction. Sci. Rep. 7, 9291 (2017). 
50. Solomon, P. S. \& Oliver, R. P. The nitrogen content of the tomato leaf apoplast increases during infection by Cladosporium fulvum. Planta 213, 241-249 (2001).

51. Solomon, P. S. \& Oliver, R. P. Evidence that $\gamma$-aminobutyric acid is a major nitrogen source during Cladosporium fulvum infection of tomato. Planta 214, 414-420 (2002).

52. Okada, T. \& Matsubara, Y. Tolerance to Fusarium root rot and the changes in free amino acid contents in mycorrhizal Asparagus plants. HortScience horts 47, 751-754 (2012).

53. Plett, J. M. et al. Effector MiSSP7 of the mutualistic fungus Laccaria bicolor stabilizes the Populus JAZ6 protein and represses jasmonic acid (JA) responsive genes. Proc. Natl. Acad. Sci. U. S. A. 111, 8299-8304 (2014).

54. Szuba, A., Marczak, Ł, Ratajczak, I., Kasprowicz-Maluśki, A. \& Mucha, J. Integrated proteomic and metabolomic analyses revealed molecular adjustments in Populus $\times$ canescens colonized with the ectomycorrhizal fungus Paxillus involutus, which limited plant host growth. Environ. Microbiol. 22, 3754-3771 (2020).

55. Maboreke, H. R. et al. Transcriptome analysis in oak uncovers a strong impact of endogenous rhythmic growth on the interaction with plant-parasitic nematodes. BMC Genom. 17, 627 (2016).

56. Rodrigues, C. I., Maia, R. \& Máguas, C. Comparing total nitrogen and crude protein content of green coffee beans (Coffea spp.) from different geographical origins. Coffee Sci. 5, 197-205 (2011).

57. Rivas-Ubach, A., Sardans, J., Pérez-Trujillo, M., Estiarte, M. \& Peñuelas, J. Strong relationship between elemental stoichiometry and metabolome in plants. Proc. Natl. Acad. Sci. 109, 4181-4186 (2012).

58. Gargallo-Garriga, A. et al. Opposite metabolic responses of shoots and roots to drought. Sci. Rep. 4, 6829 (2014).

59. Fan, T.W.-M. Metabolite profiling by one- and two-dimensional NMR analysis of complex mixtures. Prog. Nucl. Magn. Reson. Spectrosc. 28, 161-219 (1996).

60. Fan, T.W.-M. \& Lane, A. N. Structure-based profiling of metabolites and isotopomers by NMR. Prog. Nucl. Magn. Reson. Spectrosc. 52, 69-117 (2008).

61. Walker, T. E., Han, C. H., Kollman, V. H., London, R. E. \& Matwiyoff, N. A. 13C nuclear magnetic resonance studies of the biosynthesis by Microbacterium ammoniaphilum of L-glutamate selectively enriched with carbon-13. J. Biol. Chem. 257, 1189-1195 (1982).

62. Breitmaier, E. Atlas of carbon-13 NMR data. Organic Magnetic Resonanc (Springer, Berlin, 1976).

63. Iles, R. A., Hind, A. J. \& Chalmers, R. A. Use of proton nuclear magnetic resonance spectroscopy in detection and study of organic acidurias. Clin. Chem. 31, 1795-1801 (1985).

64. Bolinger L., S., S., J., K. \& J.S., L. A guide to chemical shifts of 31-P, 1-H, and 13-C. News Metab. Res. 1, 32-45 (1984).

65. Brown, J. C. C., Mills, G. A., Sadler, P. J. \& Walker, V. 1 H NMR studies of urine from premature and sick babies. Magn. Reson. Med. 11, 193-201 (1989).

66. Corse, J. \& Lundin, R. E. Diastereomers of quinic acid. Chemical and nuclear magnetic resonance studies. J. Org. Chem. 35, 1904-1909 (1970).

67. Ulrich, E. L. et al. BioMagResBank. Nucleic Acids Res. 36, D402-D408 (2007).

68. Sacchi, R., Addeo, F. \& Paolillo, L. 1H and 13C NMR of virgin olive oil. An overview. Magn. Reson. Chem. 35, S133-S145 (1997).

69. Lie Ken Jie, M. S. F. \& Lam, C. C. 1H-Nuclear magnetic resonance spectroscopic studies of saturated, acetylenic and ethylenic triacylglycerols. Chem. Phys. Lipids 77, 155-171 (1995).

70. Llusià, J., Peñuelas, J., Alessio, G. A. \& Estiarte, M. Contrasting species-specific, compound-specific, seasonal, and interannual responses of foliar isoprenoid emissions to experimental drought in a mediterranean shrubland. Int. J. Plant Sci. 169, 637-645 (2008).

71. Mangold, H. K. The Lipid Handbook. Lipid/Fett Vol. 97 (Chapman \& Hall, London, 1995).

72. The AOCS Lipid Library. (2012).

73. Maia, M. et al. Metabolite extraction for high-throughput FTICR-MS-based metabolomics of grapevine leaves. EuPA Open Proteom. 12, 4-9 (2016).

74. Kind, T. \& Fiehn, O. Seven golden rules for heuristic filtering of molecular formulas obtained by accurate mass spectrometry. BMC Bioinform. 8, 105 (2007).

75. Chong, J. et al. MetaboAnalyst 4.0: towards more transparent and integrative metabolomics analysis. Nucleic Acids Res. 46, W486W494 (2018).

76. Suhre, K. \& Schmitt-Kopplin, P. MassTRIX: mass translator into pathways. Nucleic Acids Res. 36, W481-W484 (2008).

77. Nascimento, R. et al. Early stage metabolic events associated with the establishment of Vitis vinifera - Plasmopara viticola compatible interaction. Plant Physiol. Biochem. 137, 1-13 (2019).

78. Wan, C. Y. \& Wilkins, T. A. A modified hot borate method significantly enhances the yield of high-quality RNA from cotton (Gossypium hirsutum L.). Anal. Biochem. 223, 7-12 (1994).

79. Sebastiana, M. et al. The leaf lipid composition of ectomycorrhizal oak plants shows a drought-tolerance signature. Plant Physiol. Biochem. 144, 157-165 (2019).

80. Livak, K. J. \& Schmittgen, T. D. Analysis of relative gene expression data using real-time quantitative PCR and the $2-\Delta \Delta C$ T method. Methods 25, 402-408 (2001).

81. Sebastiana, M. et al. The metabolome of an oak tree root colonized by an ectomycorrhizal fungus by FT-ICR-MS analysis. (2020). Figshare. Dataset. https://doi.org/10.6084/m9.figshare.14096213.v1

82. Sebastiana, M., Gargallo-Garriga, A., Sardans, J., Pérez-Trujillo, M. \& Peñuelas, J. Metabolic fingerprinting of the ectomycorrhizal roots of an oak tree analysed by 1H NMR. (2021). Figshare. Dataset. https://doi.org/10.6084/m9.figshare.14096213.v1

83. RStudio Team. RStudio: Integrated Development for R (RStudio, PBC, Boston, 2020).

\section{Acknowledgments}

This study was financially supported by Portuguese national funds through FCT-Fundação para a Ciência e a Tecnologia, I.P., in the context of Norma Transitória-DL57/2016/CP[12345/2018]/CT[2475] to MS, post-doc Grant SFRH/BPD/114664/2016 to FM, PhD Grant SFRH/BD/116900/2016 to MM, Investigator FCT contract IF/00819/2015 to AF and CEECIND/02246/2017 to MSS. AG was supported by the project Mobility CzechGlobe (CZ.02.2.69/0.0/0.0/16_027/0008137) and the Scholarly Studies programme of the Smithsonian Institution, project CZ.02.1.01/0.0/0.0/16_019/0000797 (SustES) of the Ministry of Education, Youth and Sports of the Czech Republic and the Research Foundation - Flanders. We also acknowledge the support from the Portuguese Mass Spectrometry Network (LISBOA-01-0145-FEDER-022125) and the Project EU_FT-ICR_MS, funded by the Europe and Union's Horizon 2020 research and innovation programme under Grant agreement nr. 731077. Work supported by UIDB/04046/2020 and UIDP/04046/2020 (to BioISI), UIDB/00329/2020 (to cE3c), UIDB/04129/2020 (to LEAF) and UIBD/00006/2020 (to CEAUL) Centre Grants from FCT, Portugal. We acknowledge the Msc student Bruno Bento for assistance with the Metaboanalyst analysis. 


\section{Author contributions}

M.S., F.M. and A.F. conceived and design the experiments. M.S. performed the mycorrhizal experiments and collected the plant material. A.G., J.S., M.P. and J.P. performed the NMR experiments and analysed the data. M.S., M.M., A.F., R.N., M.S.S., A.F., C.C. and A.P.M. performed the FT-ICR-MS analysis. M.S., M.M., A.N.F. and R.N. analysed the FT-ICR-MS data. M.S. and L.S. performed FT-ICR metabolomic statistical analysis. M.S. performed the qPCR gene expression analysis. M.S. wrote the first draft of the manuscript with the input of F.M., R.M., and J.P. All authors reviewed the manuscript.

\section{Competing interests}

The authors declare no competing interests.

\section{Additional information}

Supplementary Information The online version contains supplementary material available at https://doi.org/ 10.1038/s41598-021-87886-5.

Correspondence and requests for materials should be addressed to M.S.

Reprints and permissions information is available at www.nature.com/reprints.

Publisher's note Springer Nature remains neutral with regard to jurisdictional claims in published maps and institutional affiliations.

(c) (i) Open Access This article is licensed under a Creative Commons Attribution 4.0 International License, which permits use, sharing, adaptation, distribution and reproduction in any medium or format, as long as you give appropriate credit to the original author(s) and the source, provide a link to the Creative Commons licence, and indicate if changes were made. The images or other third party material in this article are included in the article's Creative Commons licence, unless indicated otherwise in a credit line to the material. If material is not included in the article's Creative Commons licence and your intended use is not permitted by statutory regulation or exceeds the permitted use, you will need to obtain permission directly from the copyright holder. To view a copy of this licence, visit http://creativecommons.org/licenses/by/4.0/.

(C) The Author(s) 2021 